\title{
A Comprehensive Empirical Evaluation of Generating Test Suites for Mobile Applications with Diversity
}

\author{
Thomas Vogel, Chinh Tran, Lars Grunske \\ Software Engineering Group, Humboldt-Universität zu Berlin, Berlin, Germany \\ \{thomas.vogel,grunske\}@informatik.hu-berlin.de, mail@chinhtran.de
}

\begin{abstract}
Context: In search-based software engineering we often use popular heuristics with default configurations, which typically lead to suboptimal results, or we perform experiments to identify configurations on a trial-and-error basis, which may lead to better results for a specific problem. We consider the problem of generating test suites for mobile applications (apps) and rely on SAPIENZ, a state-of-the-art approach to this problem that uses a popular heuristic (NSGA-II) with a default configuration. Objective: We want to achieve better results in generating test suites with SAPIENZ while avoiding trial-and-error experiments to identify a more suitable configuration of SAPIENZ. Method: We conducted a fitness landscape analysis of SAPIENZ to analytically understand the search problem, which allowed us to make informed decisions about the heuristic and configuration of SAPIENZ when developing SAPIENZ ${ }^{\text {div }}$. We comprehensively evaluated SAPIENZ ${ }^{d i v}$ in a headto-head comparison with SAPIENZ on 34 apps. Results: Analyzing the fitness landscape of SAPIENZ, we observed a lack of diversity of the evolved test suites and a stagnation of the search after 25 generations. SAPIENZ ${ }^{\text {div }}$ realizes mechanisms that preserve the diversity of the test suites being evolved. The evaluation showed that SAPIENZ ${ }^{\text {div }}$ achieves better or at least similar test results than SAPIENZ concerning coverage and the number of revealed faults. However, SAPIENZ ${ }^{\text {div }}$ typically produces longer test sequences and requires more execution time than SAPIEnz. Conclusions: The understanding of the search problem obtained by the fitness landscape analysis helped us to find a more suitable configuration of SAPIENZ without trial-and-error experiments. By promoting diversity of test suites during the search, improved or at least similar test results in terms of faults and coverage can be achieved.
\end{abstract}

Keywords: Fitness Landscape Analysis, Diversity, Test Generation 


\section{Introduction}

In search-based software engineering (SBSE) and particularly searchbased testing, popular heuristics (e.g., [1]) with best-practice configurations in terms of operators and parameters (e.g., [2]) are often used. As this outof-the-box usage typically leads to suboptimal results, costly trial-and-error experiments are performed to find a suitable configuration for a given problem, which leads to better results [3]. To obtain better results while avoiding trial-and-error experiments, a fitness landscape analysis can be used $[4,5]$. The goal is to analytically understand the search problem, determine difficulties of the problem, and identify suitable configurations of heuristics that can cope with these difficulties ( $c f .[4,6])$.

In previous work, we have investigated the search problem of test suite generation for mobile applications (apps) [7]. We relied on SAPIENZ [1] that uses a default NSGA-II [8] to generate test suite for apps. NSGA-II has been selected by Mao et al. [1, p. 97] as it "is a widely-used multiobjective evolutionary search algorithm, popular in SBSE research", but without adapting it to the specific problem. Thus, SAPIENZ could likely be improved by adapting NSGA-II to the problem of generating test suites for apps. For this purpose, we analyzed the fitness landscape of SAPIENZ to better understand the search problem and to make informed decisions when adapting the heuristic of SAPIENZ. With the adapted heuristic, we aimed for yielding better test results in terms of achieved coverage, revealed faults, and length of test sequences. In this context, trial-and-error experiments to empirically determine a suitable configuration of NSGA-II was not a viable option due to the high costs of executing search-based test generation approaches for apps (cf. $[7,9])$. In contrast, a fitness landscape analysis generally aims for an analytical understanding of the search problem that should help to improve the heuristic. To the best of our knowledge, our previous work [7] was the first one that analyzed the fitness landscape for the search-based generation of test suites for apps.

Our analysis of SAPIENz has focused on the global topology of the fitness landscape and evolvability, that is, how solutions (test suites) are spread in the search space and evolve over time. Thus, we are interested in the genotypic diversity of solutions being evolved, which is considered important for evolutionary search $(c f .[10])$. According to our analysis, SAPIENZ

lacks diversity of solutions so that we extended it to SAPIENZ ${ }^{\text {div }}$ that integrates four diversity-promoting mechanisms with NSGA-II. We have evalu- 
ated SAPIENZ ${ }^{d i v}$ in a preliminary evaluation in our previous work [7].

In this article extending our previous paper [7], we provide a refined discussion of the required background, fitness landscape analysis of SAPIENZ, SAPIENZ $^{\text {div }}$ approach, and related work, and particularly we present an extended empirical evaluation. This evaluation uses more apps (34 vs.10), longer runs of search ( $40 \mathrm{vs.} 10$ generations), and more repetitions of experiments (30 vs. 20) than the previous preliminary evaluation in order to conduct a comprehensive statistical analysis. To further illustrate the comprehensiveness of the extended evaluation, the corresponding experiments required 562 days of execution time (i.e., the measured wall-clock time for the whole experiment) in contrast to 32 days for the previous evaluation. The motivation to extend the search from 10 to 40 generations is that the fitness landscape analysis showed that the search of SAPIENZ stagnates after 25 generations. Thus, we can evaluate whether the diversity-promoting mechanisms of SAPIENZ ${ }^{d i v}$ will have an effect on the results when the search of SAPIEnZ actually stagnates. The evaluation shows that SAPIEnz ${ }^{\text {div }}$ achieves better or at least similar test results in terms of achieved coverage and revealed faults than SAPIENZ, but it generates fault-revealing test sequences of similar or greater length than SAPIENZ. Thus, preferring SAPIENZ ${ }^{\text {div }}$ over SAPIENZ for app testing could be advantageous concerning fault revelation and coverage, and disadvantageous concerning the length of test sequences that developers have to understand to debug the app and fix the fault in the app. Such an evaluation and discussion were not possible in our previous work due to the preliminary evaluation limiting the search to 10 generations. Therefore, the contributions of this article are the refined discussion of our work and particularly the comprehensive evaluation of SAPIENZ ${ }^{\text {div }}$ aligned with the results of the fitness landscape analysis of SAPIENZ.

The rest of the article is structured as follows. We discuss the background of our work in Section 2, the descriptive study analyzing the fitness landscape of SAPIEnZ in Section 3, SAPIENZ ${ }^{d i v}$ in Section 4, the comprehensive evaluation in Section 5, threats to validity in Section 6, and related work in Section 7. Finally, we conclude the article with Section 8.

\section{Background}

In this section, we introduce SAPIENZ and the basic idea of a fitness landscape analysis to obtain an analytical understanding of a search problem. 


\subsection{Test Suite Generation with SAPIENZ}

SAPIENZ is a multi-objective search-based testing approach [1]. Using NSGA-II, it automatically generates test suites for system-level end-to-end testing of Android apps. A test suite $t$ consists of $m$ ordered test cases $\left\langle s_{1}, s_{2}, \ldots, s_{m}\right\rangle$, each of which is a sequence of up to $n$ GUI-level events $\left\langle e_{1}, e_{2}, \ldots, e_{n}\right\rangle$ that exercise the app under test. Examples of such events are clicks and gestures.

To evolve a population of such test suites, a whole test suite variation operator is used by SAPIENZ. It consists of a uniform set element crossover among test suites applied with probability $p$ to achieve inter-individual variation, and a complex mutation operator changing single test suites to achieve intra-individual variation. This mutation operator shuffles the order of test cases within a test suite, subsequently applies a single-point crossover on two neighboring test cases of the suite with probability $q$, and finally shuffles the order of events within each test case of the suite with probability $q$. For the selection of test suites, SAPIENZ uses the select operator of NSGA-II. This select operator prefers individuals with a better fitness (smaller nondomination rank) and, when the fitness is equal, individuals with a greater diversity in the objective space (greater crowding distance). For further details of the SAPIENZ operators, we refer to [1].

The evolution of test suites is guided by three objectives: (i) maximize fault revelation, (ii) maximize coverage, and (iii) minimize test sequence length. Thus, the generated test suites should find many faults and ideally exercise the whole app while the lengths of the test sequences/cases of a suite should be minimized. Shorter test sequences are easier to be understood and reproduced by developers to identify and fix the faults revealed by them. Having no oracle, SAPIEnz considers a crash of the app caused by a test sequence as a fault. Coverage is measured at the code level (statement coverage) if the source code of the app is available, otherwise at the Android activity level (skin coverage). Given these objectives, the fitness function is the triple of the number of crashes found, achieved coverage, and sequence length. Thus, the search of SAPIENZ results in a Pareto front of test suites that are the best trade-offs with respect to the three objectives.

To evaluate the fitness of a test suite, SAPIENZ executes the suite on the app under test deployed on an Android device or emulator. In this context, SAPIENZ supports parallel evaluation of multiple test suites to improve the efficiency of the search by using several devices or emulators concurrently. 


\subsection{Fitness Landscape Analysis}

According to Culberson [11] an evolutionary algorithm is a black box as there is no clear understanding of how its operators and parameters settings interact. Therefore, "[t]he researcher trying to solve a problem is then placed in the unfortunate position of having to find a representation, operators, and parameter settings to make a poorly understood system solve a poorly understood problem. In many cases he might be better served by concentrating on the problem itself" [11, p. 125]. In this context, a fitness landscape analysis is one approach to obtain a better understanding of the search problem before making any decision about the algorithm [4]. The insights gained from the analysis should allow researchers and practitioners to make informed decisions about the algorithm to solve the problem.

According to Stadler [12], a fitness landscape is defined by three elements:

(1) A search space as a set $X$ of potential solutions.

(2) A fitness function $f_{k}: X \rightarrow \mathbb{R}$ for each of the $k$ objectives.

(3) A neighborhood relation $N: X \rightarrow 2^{X}$ that associates neighbor solutions to each solution.

The neighborhood relation is typically based on the search operators of the used algorithm that perform small changes of a solution to obtain a new solution, for instance, the mutation operator of a genetic algorithm. In this case, two solutions are neighbors if by applying the mutation operator to one solution, the other solution can be reached ( $c f .[6]$ ). According to Moser et al. [6, p. 409], it is common that the neighborhood relation is based on such local search operators since the goal is to describe fitness "landscapes where the distance between a solution and its neighbour is shorter than that between the solution and the neighbour's neighbour". Accordingly, Stadler [12] associates the notions of nearness, distance, or accessibility to the neighborhood relation. Thus, it is possible to quantify the neighborhood relation using a distance metric that is based on the local search operators of the genetic algorithm being used. Relying on a search operator of an algorithm for the neighborhood relation, each operator describes an individual fitness landscape, which is called "one operator, one landscape" by Jones [13]. Therefore, the fitness landscape can be seen as a description of the (1) search space as explored by the genetic algorithm that uses the (2) fitness functions for guiding the search and whose operators determine the (3) neighborhood relation. 
Given these three elements defining a fitness landscape, various metrics have been proposed to analyze the landscape $[4,5]$. They characterize the landscape, for instance, in terms of the global topology (i.e., the distributions of solutions and fitness within the landscape), local structure (i.e., the number and distribution of local optima, the shape of landscape areas in terms of ruggedness and smoothness, or plateaus as connected areas of equal fitness), and evolvability (i.e., the ability to produce fitter solutions denoting a progress of the search). The goal of analyzing the landscape is to determine difficulties of a search problem and identify suitable configurations of search algorithms that can cope with these difficulties (cf. $[4,6])$. For this purpose, the fitness landscape analysis opens the black box of a genetic algorithm and investigates how the algorithm explores the search space.

\section{Fitness Landscape Analysis of SAPIENZ}

In this section, we discuss our descriptive study analyzing the fitness landscape of SAPIENZ. For this purpose, we define the fitness landscape of SAPIENZ in Section 3.1 and present the design, experimental setup, and results of analyzing the fitness landscape of SAPIENZ in Sections 3.2 and 3.3. Finally, we discuss these results in Section 3.4.

\subsection{Fitness Landscape of SAPIENZ}

First, we define the fitness landscape of SAPIENZ in terms of the three elements of a fitness landscape ( $c f$. Section 2.2):

(1) The search space of SAPIENZ is given by all possible test suites $t$ according to the representation of test suites in SAPIENZ (Section 2.1).

(2) The fitness function is given by the triple of the number of crashes found, achieved coverage, and test sequence length (Section 2.1).

(3) As the neighborhood relation we define the metric $\operatorname{dist}\left(t_{1}, t_{2}\right)$ that computes the distance between two test suites $t_{1}$ and $t_{2}$ at the genotypic level.

The distance metric $\operatorname{dist}\left(t_{1}, t_{2}\right)$ (Algorithm 1) has as input two parameters, the test suites $t_{1}$ and $t_{2}$ as produced by SAPIENZ, whereas the test suite size $m$ and the maximal test sequence length $n$ are determined by the static configuration of SAPIENZ. The metric computes the genotypic distance between $t_{1}$ and $t_{2}$, which is the output of the algorithm, as follows. The distance between two test suites $t_{1}$ and $t_{2}$ is the sum of the distances between 


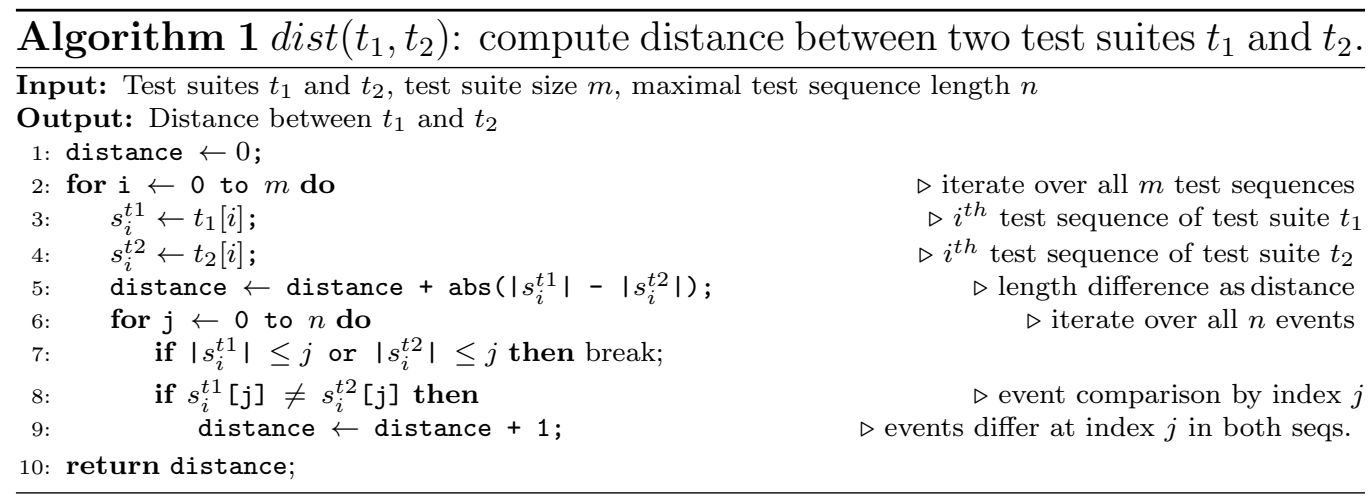

their ordered test sequences, which is obtained by comparing all sequences $s_{i}^{t 1}$ of $t_{1}$ and $s_{i}^{t 2}$ of $t_{2}$ by index $i$ (lines $2-4$ ). The distance between two test sequences $s_{i}^{t 1}$ and $s_{i}^{t 2}$ is the difference of their lengths (line 5) increased by 1 for each different event at index $j$ (lines 6-9). Thus, the distance is based on the differences of ordered events between the ordered test sequences of two test suites.

Since it is common that the neighborhood relation is based on the search operators of the genetic algorithm being used that make small changes to solutions ( $c f$. Section 2.2), we developed this distance metric according to the mutation operator of SAPIENZ that performs small changes on individual test suites. The smallest changes performed by mutation are the shuffling of the order of test sequences within a test suite, and the order of events within a test sequence. Consequently, to align the neighborhood relation for the fitness landscape to the search operators of SAPIENZ, we defined a corresponding distance metric rather than using a standard edit distance.

\subsection{Design and Experimental Setup of the Fitness Landscape Analysis}

Among the global topology, local structure, and evolvability that can be investigated by a fitness landscape analysis (Section 2.2), we are interested in the global topology, that is, how solutions (test suites) are spread in the search space during the search process of SAPIEnz. Thus, we investigate the genotypic diversity of the solutions being evolved since it is known that diversity has an influence on the performance of genetic algorithms ( $c f .[10])$. For instance, Panichella et al. [14] have shown in the context of regression testing that the selection of test cases based on genetic algorithms could be improved by diversifying the test cases during the search. However, the diversity of solutions in the context of generating test suites for apps has not 
Table 1: Apps and their test results [1] we selected for the fitness landscape analysis.

\begin{tabular}{lclccc}
\hline Name & Version & Category & Coverage (\%) & \#Crashes & Seq. Length \\
\hline aarddict & 1.4 .1 & Books \& Reference & 18 & 0 & - \\
passwordmanager & 1.9 .8 & Tools & 16 & 0 & - \\
MunchLife & 1.4 .2 & Entertainment & 76 & 0 & - \\
hotdeath & 1.0 .7 & Card & 79 & 3 & 152 \\
k9mail & 5.207 & Communications & 7 & 1 & 238 \\
\hline
\end{tabular}

been investigated yet by a fitness landscape analysis. Besides the diversity of the test suites during the search, we investigate the evolvability, that is, the progress of the search and how the test suites evolve over time.

To analyze the diversity and evolution of test suites in SAPIENZ, we selected 11 different metrics from literature since "[n]o single measure or description can possibly characterize any high-dimensional heterogeneous search space" $[15$, p. 31] or the difficulty of the related search problems $[4,5]$. This view holds for SAPIENZ and the generation of test suites for apps representing such a complex search space and problem. These 11 metrics characterize the evolvability of the search and the diversity of the whole population and Pareto-optimal solutions. We implemented them in SAPIENZ and compute them after every generation of the search process so that we can analyze their development over time, and thus, the progress of the search and how the solutions and their diversity evolve. We will discuss each metric when presenting the results of the fitness landscape analysis in the next section.

For the fitness landscape analysis of SAPIEnz, we set up an experiment. Due to the high execution costs of SAPIENZ, we selected five apps (Table 1) from the 68 F-Droid apps benchmark [16] used to evaluate SAPIEnz [1]. Particularly, we selected aarddict, passwordmanager, and MunchLife since SAPIENZ did not find any fault for them, and hotdeath and $k 9$ mail $^{1}$ for which SAPIENZ did find faults [1]. Moreover, the individual apps are from different app categories and SAPIENZ achieved different levels of statement coverage and generated fault-revealing test sequences of different lengths for them (Table 1). Thus, we consider apps with different test results to obtain potentially different fitness landscape features that may present difficulties to the SAPIENZ search.

\footnotetext{
${ }^{1}$ We used version 5.207 of k9mail instead of 3.512 as in the 68 F-Droid apps benchmark [16] due to unavailability of the respective version at the time of study. The other four apps are of the same version as in the benchmark.
} 
Table 2: SAPIEnZ configuration.

\begin{tabular}{lr}
\hline Parameter & Value \\
\hline Crossover rate & 0.7 \\
Mutation rate & 0.3 \\
Population size & 50 \\
Offspring size & 50 \\
Test suite size (number of test cases per test suite) & 5 \\
Minimum length of a test sequence (number of events) & 20 \\
Maximum length of a test sequence (number of events) & 500 \\
Number of generations (stopping criterion of the search) & 40 \\
\hline
\end{tabular}

To conduct the experiment, we execute SAPIENZ extended with the 11 metrics on each of the five selected apps, repeat each execution five times, and report for each app the time series (over the generations) of the metric values averaged over the repetitions. ${ }^{2}$ We use the default configuration of SAPIENZ [1] listed in Table 2. The crossover and mutation rates are set to 0.7 and 0.3 respectively. The population size and offspring size are 50 . An individual (test suite) contains five test sequences, each constrained to 20-500 events. However, we set the stopping criterion of the search to 40 generations to obtain longer runs than in [1].

\subsection{Results of the Fitness Landscape Analysis}

The results of our descriptive study provide an analysis of the fitness landscape of SAPIENZ with respect to the evolvability, that is, the progress of the search, and global topology, that is, the diversity and spread of solutions (test suites) in the search space. In the following, we present these results by discussing the 11 metrics we implemented in SAPIENZ and the time series we obtained for these metrics by executing SAPIENZ on each of the five selected apps. The discussion is grouped by the purpose of the metrics as they characterize either (1) the evolvability of the search, (2) the diversity of the whole population, or (3) the diversity of Pareto-optimal solutions.

\subsubsection{Metrics for the Evolvability of the Search}

The following two metrics characterize the evolvability of the search by looking at the evolution of the Pareto-optimal solutions during the search.

\footnotetext{
${ }^{2}$ All experiments were run on a single $4.0 \mathrm{GHz}$ quad-core PC with $16 \mathrm{~GB}$ RAM, using five Android emulators (KitKat 4.4.2, API level 19) in parallel to test one app.
} 


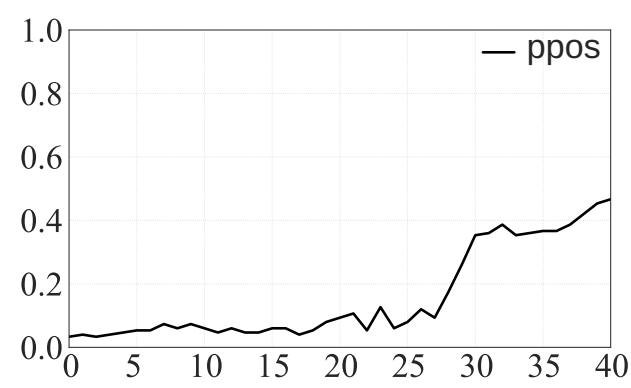

(a) aarddict

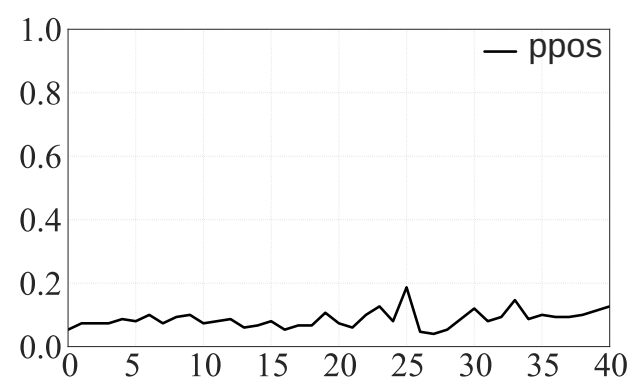

(c) passwordmanager

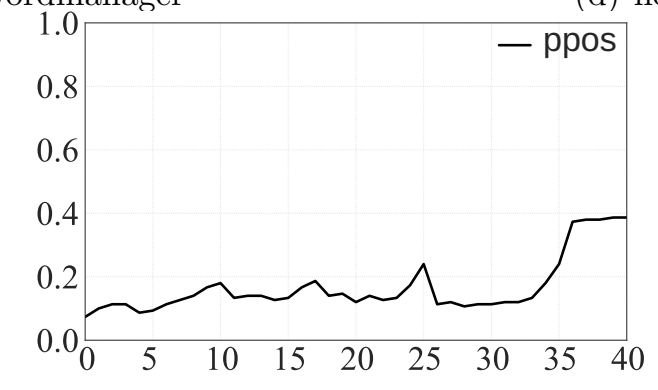

(e) k9mail

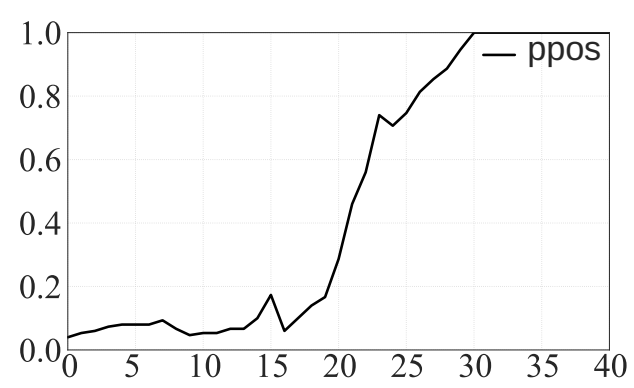

(b) MunchLife

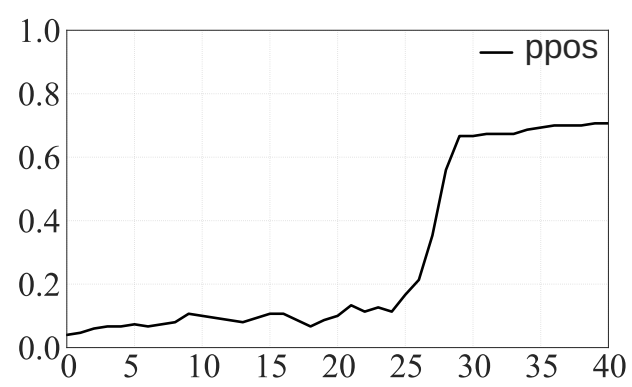

(d) hotdeath

Figure 1: Proportion of Pareto-optimal solutions (ppos) over the 40 generations of search.

Proportion of Pareto-optimal solutions (ppos). For a population $P$, ppos is the number of Pareto-optimal solutions $P_{o p t}$ (i.e., the non-dominated solutions of $P$ ) divided by the population size: $\operatorname{ppos}(P)=\frac{\left|P_{\text {opt }}\right|}{|P|}$. A high and especially strongly increasing ppos may indicate a problem of dominance resistance, that is, the search cannot produce new solutions by crossover and mutation that dominate the current, poorly performing but locally nondominated solutions. Due to this missing selection pressure, the search based on Pareto dominance may stagnate, potentially far away from the true Pareto front [17]. A moderately increasing ppos may indicate a successful search.

For SAPIENZ and all apps (see Fig. 1 plotting ppos averaged over the five 
runs along the 40 generations of the search for each of the five apps), ppos slightly fluctuates since a single new solution from the current generation can potentially dominate multiple solutions that have been non-dominated in the previous generation. In this case, ppos decreases. In contrast, ppos increases if more new solutions are found than such previously non-dominated solutions that are now dominated by the new solutions, or if new solutions just extend the Pareto front without dominating any other non-dominated solution of the previous generation. At the beginning of the search, ppos is low $(<0.1)$, shows no improvement in the first 15-20 generations, and then increases for all apps except of passwordmanager. Thus, the search seems to progress while the enormously increasing ppos for MunchLife and hotdeath after around 20 and 25 generations respectively might indicate a stagnation of the search.

Hypervolume (hv). To further investigate the search progress, we compute the $h v$ after each generation. The $h v$ is the volume in the objective space covered by the Pareto-optimal solutions [18, 19]. According to Li and Yao [18], it is the most popular quality indicator in optimization and used to compare different Pareto fronts or generally, two sets of solutions. If one set is better than the other with respect to the objectives of the search, then the $h v$ of the better set is higher than the $h v$ of the other set. Thus, within a search process, an increasing $h v$ indicates that the search is able to find improved solutions, otherwise the $h v$ and search stagnate.

Based on the objectives of SAPIENZ (i.e., maximize the number of crashes, maximize the achieved coverage, and minimize the length of test sequences), we choose the nadir point as the reference point for the $h v$, that is, 0 crashes, 0 coverage, and a test sequence length of 500 (we configured SAPIENZ to generate test sequences that consists of at most 500 events ( $c f$. Section 3.2)).

In Fig. 2, the evolution of the $h v$ over time rather than the absolute numbers are relevant to analyze the search progress of SAPIEnz. While the $h v$ increases during the first 25 generations, it stagnates afterwards for all apps; for k9mail already after 5 generations. Thus, the search of SAPIENZ is able to find improved solutions but it stagnates after 25 generations. For aarddict, MunchLife, and hotdeath the $h v$ stagnates after the ppos drastically increases ( $c f$. Fig. 1), further indicating a stagnation of the search after 20-25 generations. 


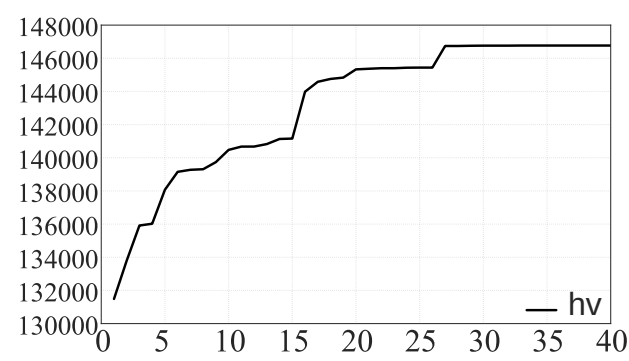

(a) aarddict

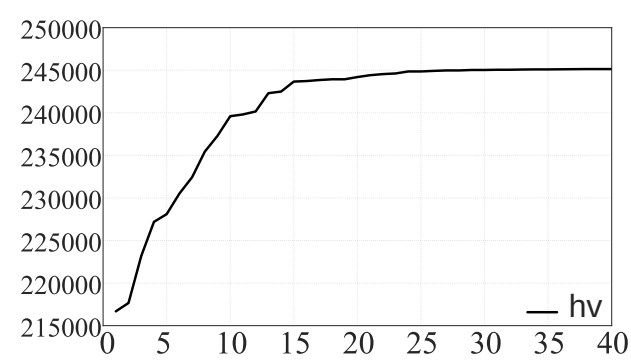

(c) passwordmanager

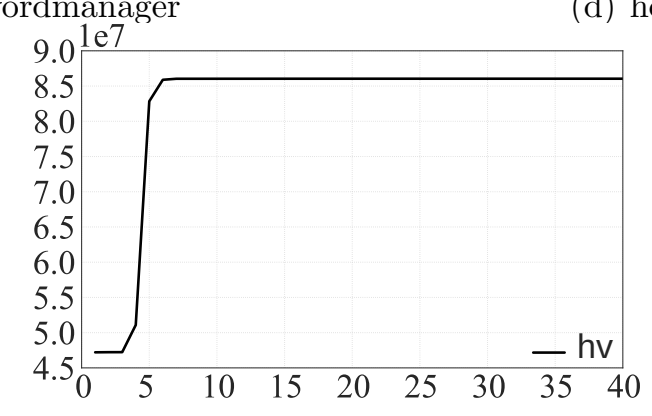

(e) k9mail

Figure 2: Hypervolume ( $h v)$ over the 40 generations of search.

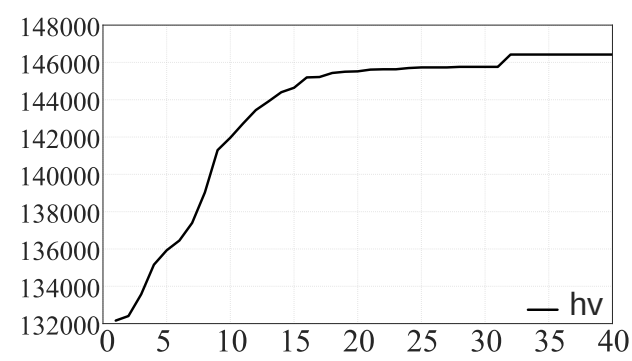

(b) MunchLife

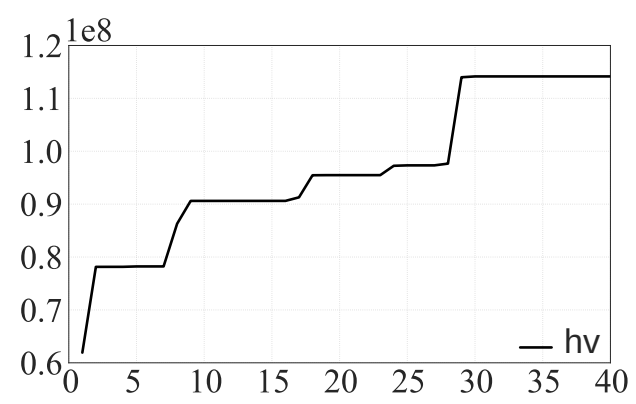

(d) hotdeath 


\subsubsection{Metrics for the Diversity of the Population}

The following four metrics characterize the diversity of the whole population, that is, of all individuals in each generation of the search.

Maximum, Average, and Minimum Population diameter (maxdiam, avgdiam, mindiam). These metrics measure the spread of all population members in the search space using a distance metric for individuals. In our case, we use the metric $\operatorname{dist}\left(t_{1}, t_{2}\right)$ defined by Algorithm 1 computing the distance between any two test suites $t_{1}$ and $t_{2}$. The maximum population diameter computes the largest distance between any two individuals of the population $P: \operatorname{maxdiam}(P)=\max _{x_{i}, x_{j} \in P} \operatorname{dist}\left(x_{i}, x_{j}\right)$ [20, 21]. Thus, maxdiam shows the absolute spread of $P$ in the search space. To respect outliers, we can compute the average population diameter as the average of all pairwise distances between all individuals [20]:

$$
\operatorname{avgdiam}(P)=\frac{\sum_{i=0}^{|P|} \sum_{j=0, j \neq i}^{|P|} \operatorname{dist}\left(x_{i}, x_{j}\right)}{|P|(|P|-1)}
$$

Additionally, we compute the minimum population diameter to see how close individuals are in the search space: $\operatorname{mindiam}(P)=\min _{x_{i}, x_{j} \in P} \operatorname{dist}\left(x_{i}, x_{j}\right)$. Using mindiam, we can test whether individuals are even identical and thus represent duplicate solutions in a population. In this case, mindiam is 0 .

Concerning each plot in Fig. 3, the upper, middle, and lower curve are respectively maxdiam, avgdiam, and mindiam. Since all of these three metrics rely on our distance metric $\operatorname{dist}\left(t_{1}, t_{2}\right)$, their ranges are determined by the distance metric that ranges from 0 (the two solutions $t_{1}$ and $t_{2}$ are identical) to 2500 (the two solutions $t_{1}$ and $t_{2}$ differ as much as possible, that is, they differ in all events - up to 500 events for a test sequence - for all of their five individual test sequences). For each curve, we see a clear trend that the metrics decrease over time, which is typical for genetic algorithms due to the crossover. However, the metrics drastically decrease for SAPIENZ in the first 25 generations. The avgdiam decreases from $>1500$ to eventually $<200$ for each app. The maxdiam decreases similarly from around 2250 to $\approx 250$ but stays higher for hotdeath $(>500)$ and k9mail $(\approx 1000)$, which suggests that there are a few outliers in these two apps. The development of the avgdiam and maxdiam indicates that all individuals are continuously getting closer to each other in the search space, thus becoming more similar. The population even contains identical solutions as indicated by mindiam reaching 0 . 


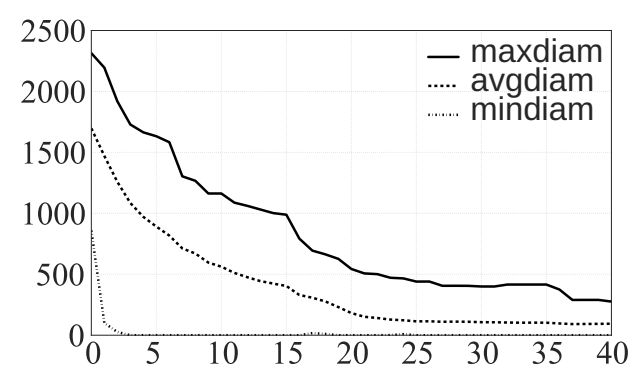

(a) aarddict

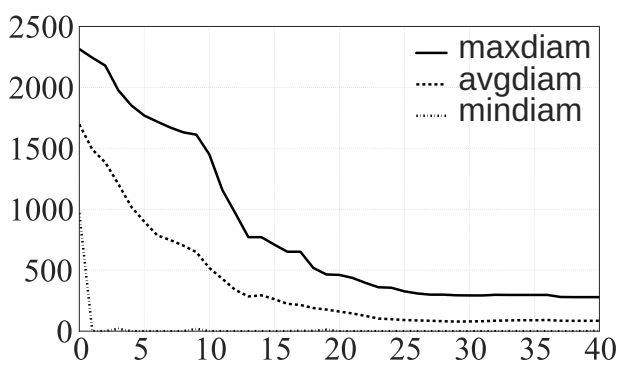

(c) passwordmanager

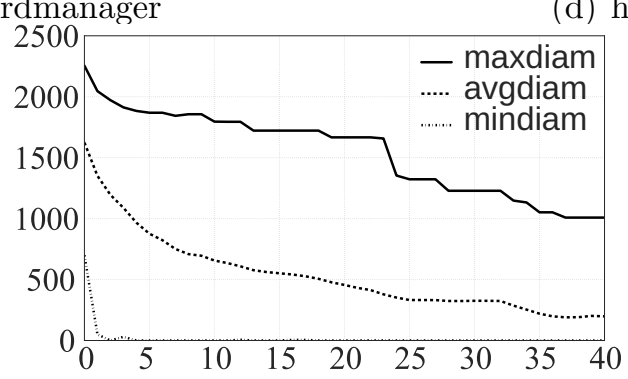

(e) k9mail

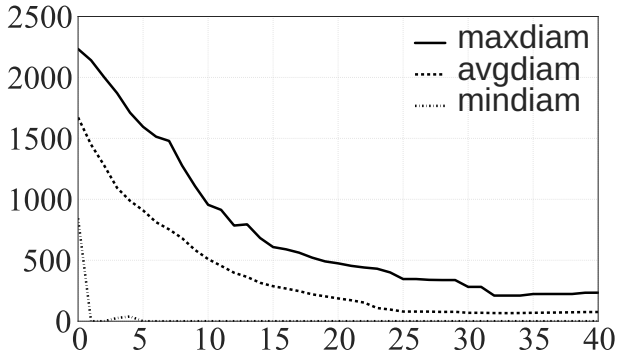

(b) MunchLife

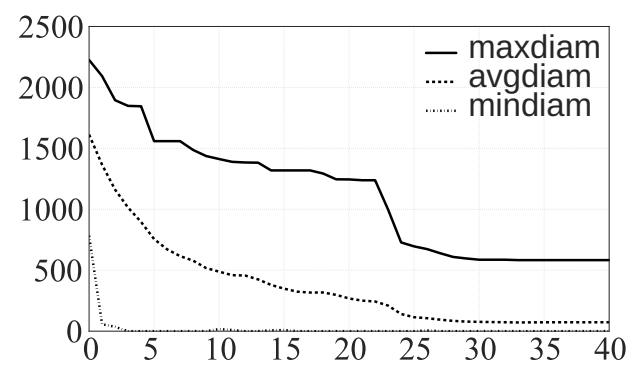

(d) hotdeath

Figure 3: Maximum, average, and minimum population diameters (maxdiam, avgdiam, mindiam) over the 40 generations of search.

Relative population diameter (reldiam). Besides the population diameter metrics discussed previously, Bachelet [20] further proposes the relative population diameter (reldiam), which is the average population diameter (avgdiam) in proportion to the largest possible distance $d$ : $\operatorname{reldiam}(P)=\frac{\operatorname{avgdiam}(P)}{d}$. This metric is indicative of the concentration of the population in the search space. Especially, a small reldiam indicates that the population members are grouped together in a region of the space [20].

For SAPIEnZ, the largest possible distance $d$ between two test suites is 2500 , in which case they differ in all events (up to 500 for a test sequence) for all of their five individual test sequences ( $c f$. previous discussion of the 
diameter metrics). For $d=2500$ and all apps ( $c f$. Fig. 4), reldiam starts at a high level of around 0.9 indicating that the solutions are spread in the search space. Then, it decreases in the first 25 generations to around 0.4 for aarddict, MunchLife, and passwordmanager, and below 0.3 for hotdeath and k9mail indicating a grouping of the solutions in a region of the search space. Thus, we observe a continuous concentration process of the population in the search space in the first 25 generations, after which the concentration stays high for aarddict, hotdeath, and k9mail (reldiam is relatively constant), or slightly decreases for MunchLife and passwordmanager (reldiam slightly increases).

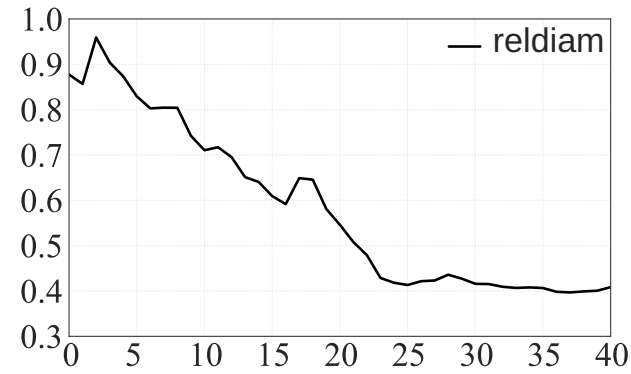

(a) aarddict

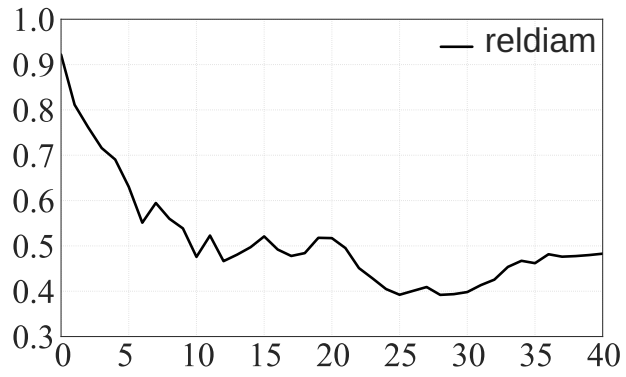

(c) passwordmanager

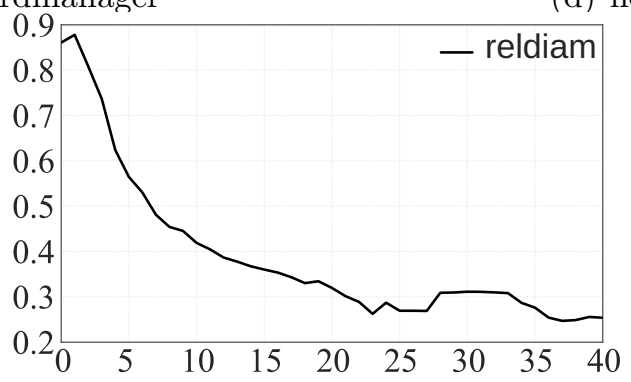

(e) k9mail

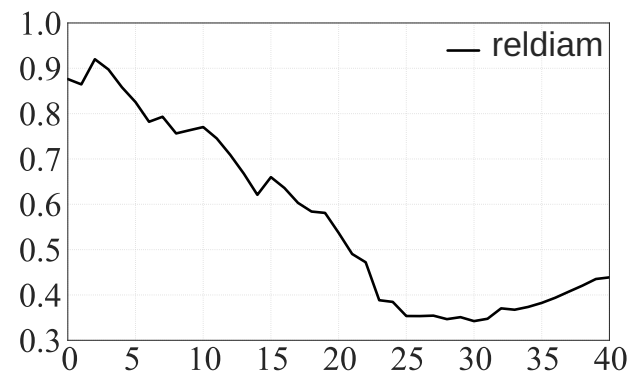

(b) MunchLife

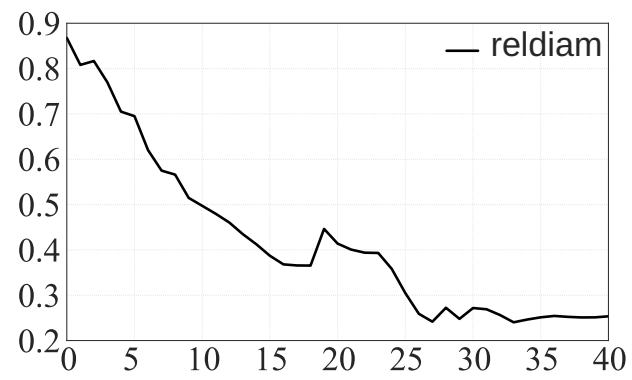

(d) hotdeath

Figure 4: Relative population diameter (reldiam) over the 40 generations of search. 


\subsubsection{Metrics for the Diversity of the Pareto-Optimal Solutions}

The following metrics analyze the connectedness and thus, clusters of Pareto-optimal solutions in the search space [22, 23]. For this purpose, we consider a graph in which Pareto-optimal solutions are vertices. The edges connecting the vertices depend on the adjacency of Pareto-optimal solutions defined by a neighborhood operator. Particularly, the edges are labeled with weights $\delta$, which are the number of moves a neighborhood operator has to make to reach one vertex from another [23]. This results in a graph of fully connected Pareto-optimal solutions. Introducing a limit $k$ on $\delta$ and removing the edges whose weights $\delta$ are larger than $k$ leads to varying sizes of connected components (clusters) in the graph. This graph can be analyzed by metrics to characterize the Pareto-optimal solutions in the search space [23, 24].

In our case, the weights $\delta$ are determined by the distance metric $\operatorname{dist}\left(t_{1}, t_{2}\right)$ for two test suites $t_{1}$ and $t_{2}(c f$. Algorithm 1$)$ that is based on the mutation operator of SAPIENZ ( $c f$. Section 3.1). We determined $k$ experimentally to be 300 investigating values of 400, 300, 200, and 100. While a high value results in a single cluster of Pareto-optimal solutions, a low value results in a high number of singletons (i.e., many clusters each containing a single Paretooptimal solution). Thus, two test suites (vertices) are connected (neighbors) in the graph if they differ in less than 300 events across their five individual test sequences as computed by the distance metric dist $\left(t_{1}, t_{2}\right)$. Based on such a graph of Pareto-optimal solutions for SAPIEnz, we compute in each generation of the search the following five metrics to analyze the Pareto-optimal solutions, their connectedness, and the corresponding evolution over time.

Proportion of Pareto-optimal solutions in clusters (pconnec). This metric divides the number of vertices (Pareto-optimal solutions) that are members of clusters (excluding singletons) by the total number of vertices in the graph [23]. A high pconnec indicates a grouping of the Pareto-optimal solutions in one or more areas of the search space while a low value indicates a spread of these solutions across the search space.

As shown in Fig. 5, pconnec is relatively low during the first generations before it increases for all apps. For MunchLife, passwordmanager, and hotdeath, pconnec reaches 1 meaning that all Pareto-optimal solutions are located in (one or more) clusters, while it converges around 0.7 and 0.8 for aarddict and k9mail respectively. Overall, this indicates that all or at least most of the Pareto-optimal solutions are grouped in one or more areas of the search space. 


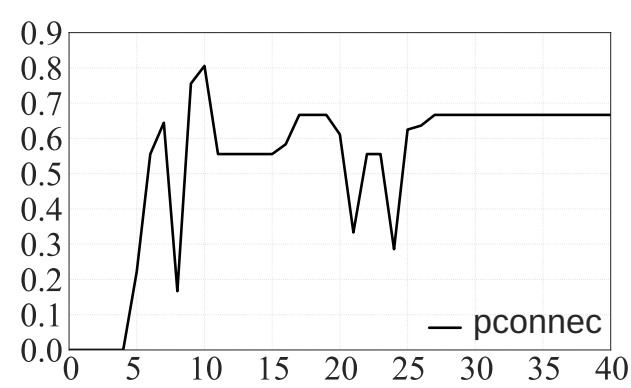

(a) aarddict

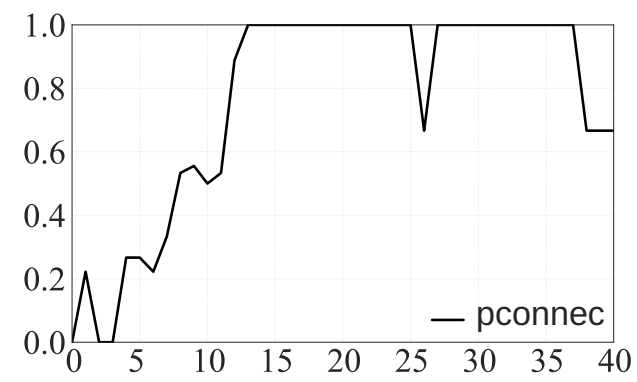

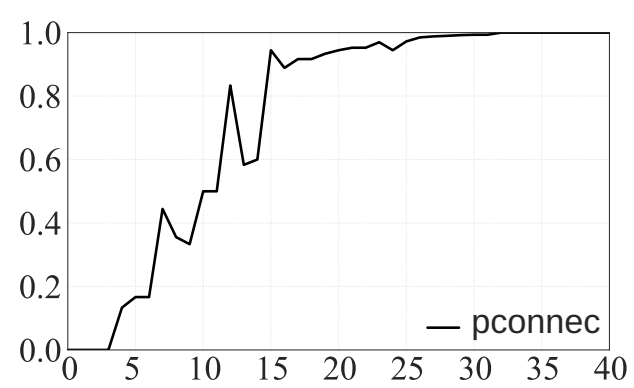

(b) MunchLife

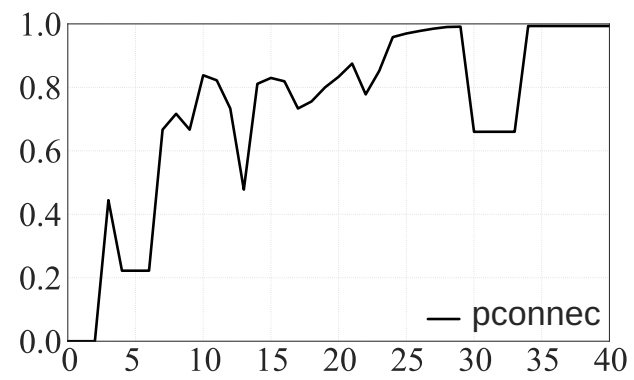

(d) hotdeath

(c) passwordmanager

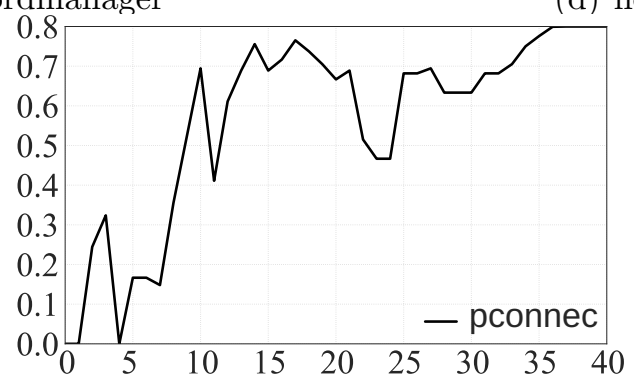

(e) k9mail

Figure 5: Proportion of Pareto-optimal solutions in clusters (pconnec) over the 40 generations of search.

Number of clusters (nconnec). We further analyze in how many areas of the search space (clusters) the Pareto-optimal solutions are grouped. Thus, nconnec counts the number of clusters in the graph [23, 24]. A high nconnec indicates that the Pareto-optimal solutions are spread in many areas, whereas a low nconnec indicates that the Pareto-optimal solutions are spread in few areas of the search space.

Fig. 6 plots nconnec for SAPIENZ and all apps. The y-axis of each plot denoting nconnec ranges from 0 to 6 . Initially, the Pareto-optimal solutions are distributed in 2-4 clusters, then grouped in 1 cluster. A noticeable exception is k9mail for which there always exist more than 3 clusters. Except 


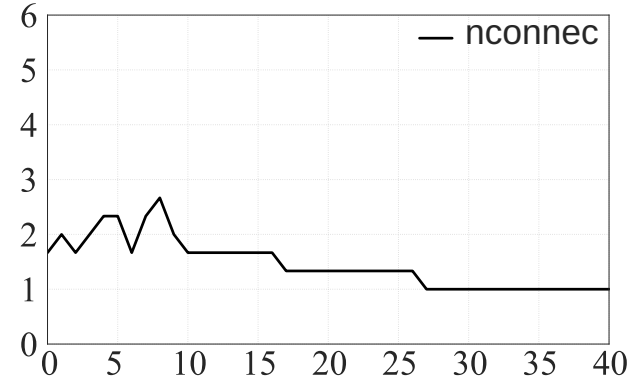

(a) aarddict

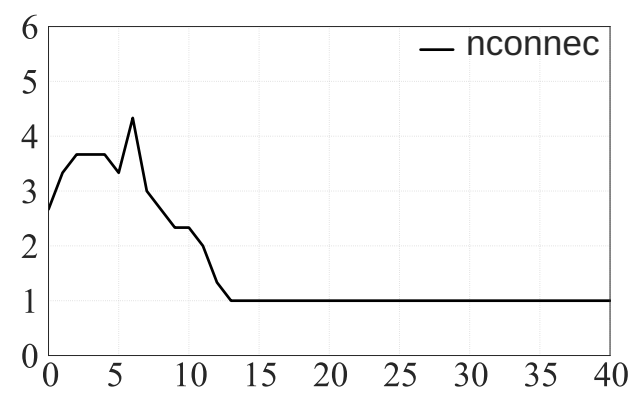

(c) passwordmanager

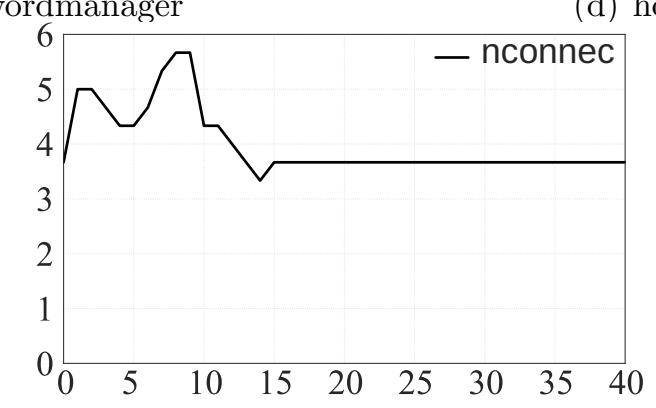

(e) k9mail

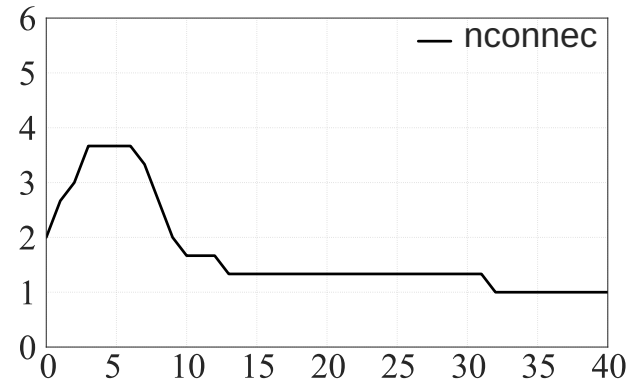

(b) MunchLife

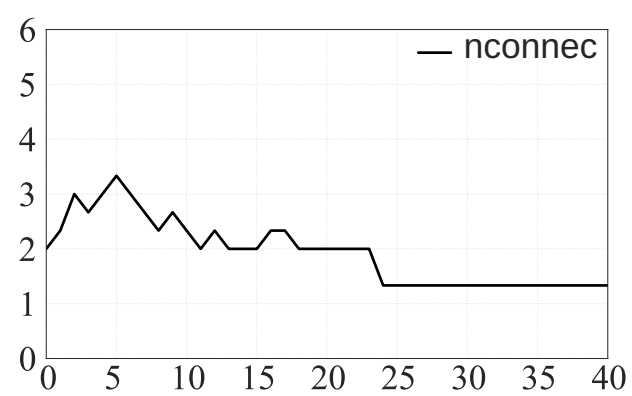

(d) hotdeath

Figure 6: Number of clusters (nconnec) over the 40 generations of search.

for $\mathrm{k} 9$ mail, this indicates that the Pareto-optimal solutions are grouped in one area of the search space as they belong to one cluster.

Minimum distance $k$ for a connected graph (kconnec). This metric identifies the limit $k$ ( $c f$. first paragraph in Section 3.3.3) so that all Pareto-optimal solutions are members of one cluster $[23,24]$. In this case, the single cluster covers the whole graph. In other words, the whole graph is connected. Thus, kconnec quantifies the spread of all Pareto-optimal solutions in the search space.

For SAPIENZ, Fig. 7 plots kconnec (ranging from 0 to 1400) over the gen- 


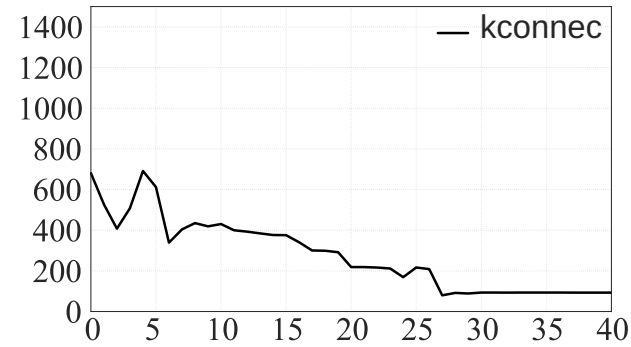

(a) aarddict

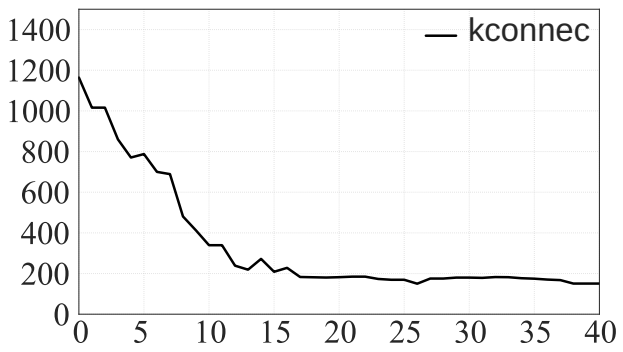

(c) passwordmanager

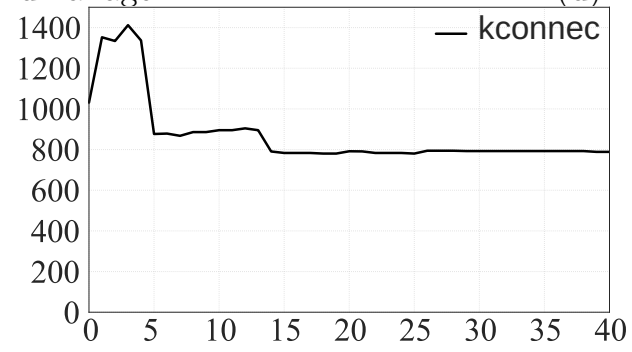

(e) k9mail

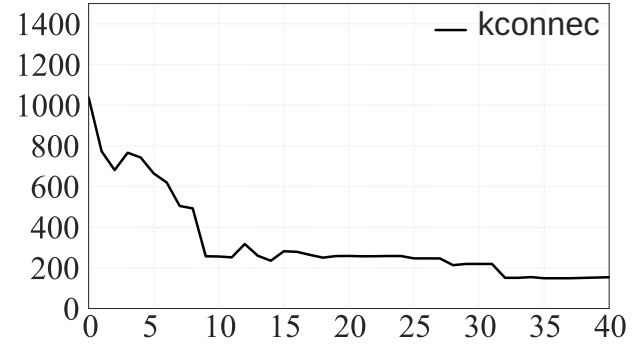

(b) MunchLife

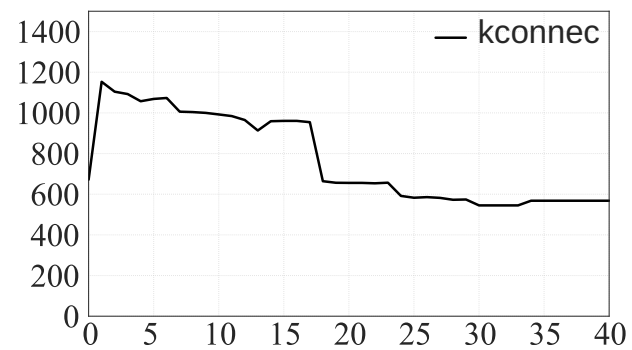

(d) hotdeath

Figure 7: Minimum distance $k$ for a connected graph (kconnec) over the 40 generations of search.

erations. Similarly to the diameter metrics (cf. Fig. 3), kconnec decreases, moderately for hotdeath (from initially $\approx 700$ to $\approx 600$ in the first 25 generations) and $\mathrm{k} 9$ mail (from $\approx 1000$ to $\approx 800$ in the first 15 generations), and drastically for passwordmanager (from $\approx 1200$ to $\approx 200$ in the first 15 generations), MunchLife (from $\approx 1000$ to $\approx 200$ in the first 30 generations), and aarddict (from $\approx 600$ to $\approx 100$ in the first 27 generations). Afterwards, kconnec stays relatively constant around its low individual level for each app.

This indicates that all Pareto-optimal solutions are getting closer to each other in the search space as the spread of the connected graph is decreasing while this process is converging. 
Number of Pareto-optimal solutions in the largest cluster (lconnec). This metric determines the size of the largest cluster by the number of its members [24], showing how many Pareto-optimal solutions are located in the most dense area of the search space. Thus, this metric counts the number of vertices of the graph that are members of the largest cluster.

Fig. 8 plots lconnec ranging from 1 to 50 given the population size of 50 (cf. Section 3.2) over the generations. lconnec increases after 15-30 generations to 20 (aarddict and hotdeath) or even 50 (MunchLife) solutions. This indicates that the largest cluster is indeed large so that many Pareto-optimal solutions are grouped in one area of the search space. In contrast, lconnec

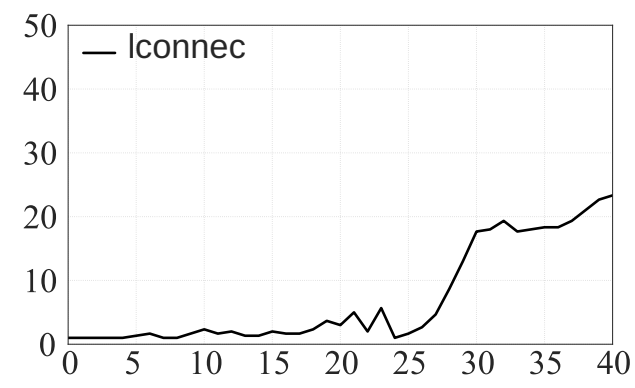

(a) aarddict

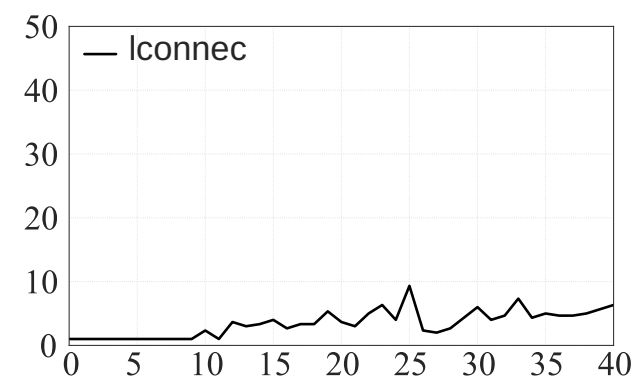

(c) passwordmanager

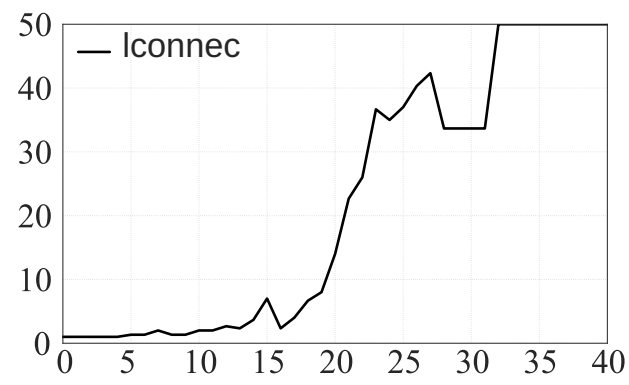

(b) MunchLife

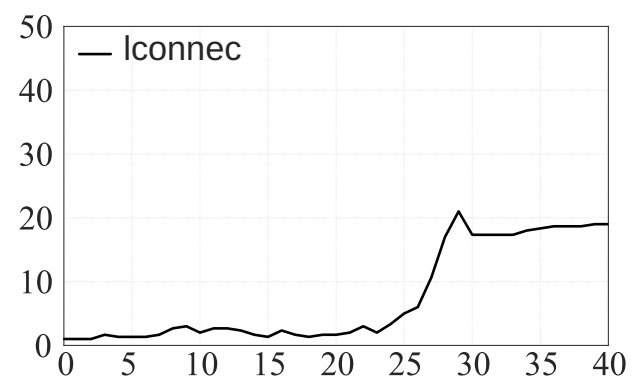

(d) hotdeath

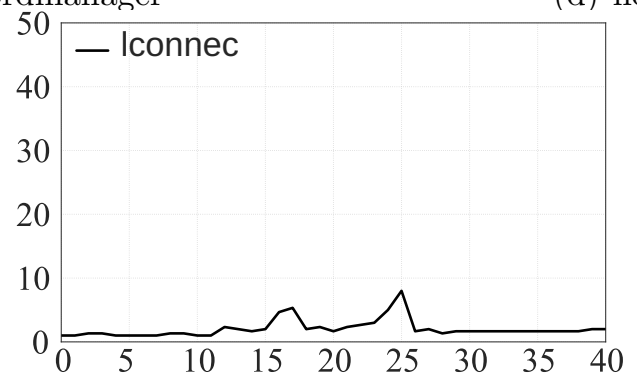

(e) k9mail

Figure 8: Number of Pareto-optimal solutions in the largest cluster (lconnec) over the 40 generations of search. 
stays always below 10 indicating smaller largest clusters for passwordmanager and k9mail than for the other apps.

lconnec basically follows the trend of the proportion of Pareto-optimal solutions (ppos) in Fig. 1 since a low ppos automatically leads to a low number of vertices in the graph (Pareto-optimal solutions) and thus, also a lower number of such solutions in the largest cluster.

Proportion of hypervolume covered by the largest cluster (hvconnec). Besides the size of the largest cluster of the graph in terms of number of solutions (lconnec), we compute the relative size of the largest cluster in terms of

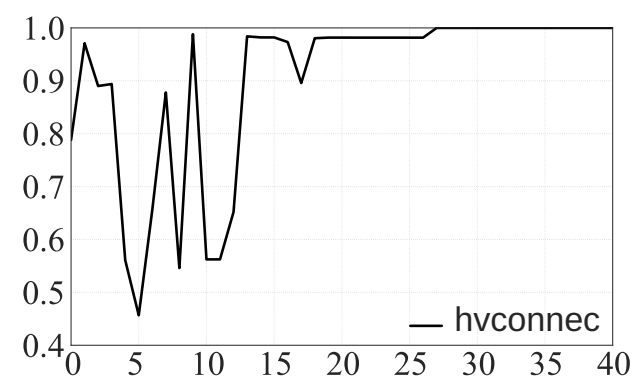

(a) aarddict

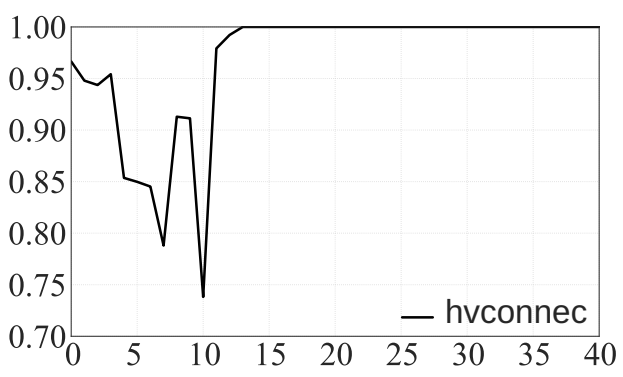

(c) passwordmanager

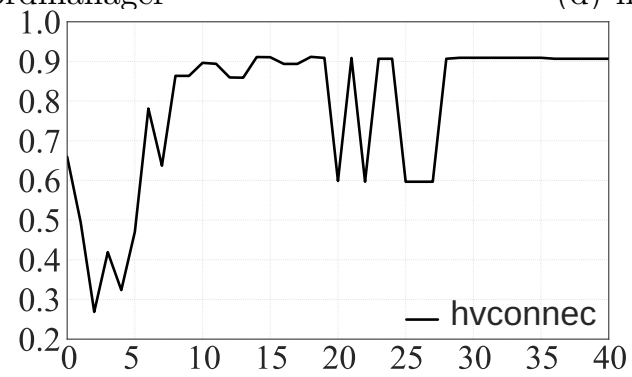

(e) k9mail

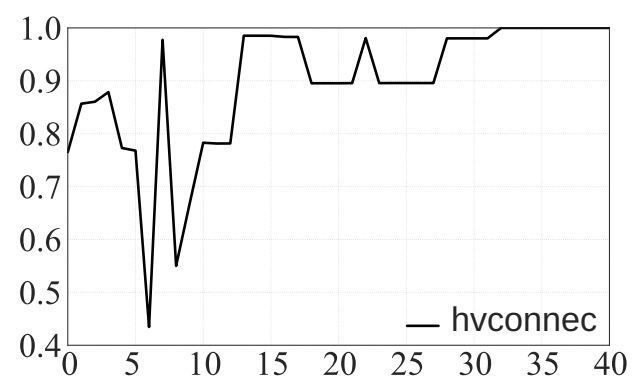

(b) MunchLife

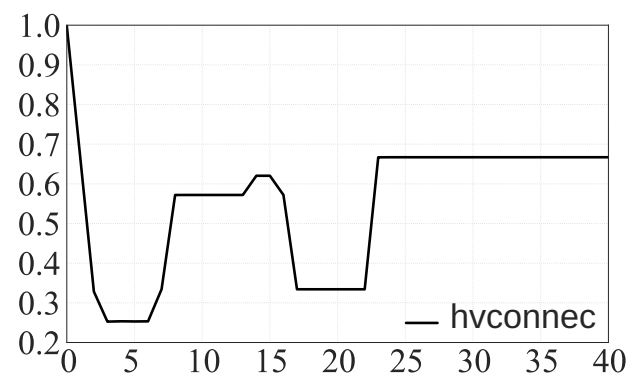

(d) hotdeath

Figure 9: Proportion of hypervolume covered by the largest cluster (hvconnec) over the 40 generations of search. 
hypervolume $(h v)$. Thus, hvconnec is the proportion of the overall $h v$ covered by the Pareto-optimal solutions in the largest cluster. It quantifies how the largest cluster in the search space dominates in the objective space and contributes to the overall $h v$. The higher hvconnec, the more the largest cluster contributes to the $h v$.

For SAPIEnZ ( $c f$. Fig. 9), hvconnec varies a lot during the first 10 generations, then stabilizes at a high level for all apps. For aarddict, MunchLife, and passwordmanager, the largest clusters covers $100 \%$ of the $h v$ since there is only one cluster left ( $c f$. nconnec in Fig. 6). For hotdeath, hvconnec is close to $70 \%$ indicating that there is one other cluster covering $30 \%$ of the $h v$ (cf. nconnec for hotdeath). For k9mail, hvconnec is around $90 \%$ indicating that the other 2-3 clusters ( $c f$. nconnec for k9mail) cover only $10 \%$ of the

$h v$. This indicates that the largest cluster covers the largest proportion of the $h v$, and thus contributes most to the Pareto front.

\subsection{Discussion}

The results characterizing the fitness landscape of SAPIENZ that we presented in the previous sections reveal insights about how SAPIENZ manages the search problem of generating test suites for apps. A summary of these insights gained by each of the 11 metrics is provided by Table 3 .

Firstly, the strongly increasing proportion of Pareto-optimal solutions (ppos) for some apps may indicate a problem of dominance resistance, that is, the search cannot produce new solutions by crossover and mutation that dominate the current, poorly performing but locally non-dominated solutions [17]. In other cases, ppos remains low, that is, the search cannot find many non-dominated solutions. Thus, ppos may indicate a stagnation of the search, which is witnessed by the stagnation of the hypervolume $(h v)$ after 25 generations of search. Consequently, the evolvability of SAPIEnz as the ability to produce test suites with better fitness declines after 25 generations.

Secondly, the development of the maximum population diameter (maxdiam) indicates a decreasing maximum spread of the population in the search space as determined by the population's two most distant individuals. Furthermore, the development of the average population diameter (avgdiam) shows a decreasing diversity of all individuals during the search. The population even contains duplicates of individuals, which reduce the genetic variation in the population, as indicated by the minimum population diameter (mindiam). The development of the relative population diameter (reldiam) witnesses this observation and indicates that the population members are 
Table 3: Summary of the metrics and insights of the fitness landscape analysis of SAPIENZ.

\begin{tabular}{|c|c|c|c|}
\hline \# & Metric & General description & Insights for SAPIENZ \\
\hline \multicolumn{4}{|c|}{ Metrics for the evolvability of the search } \\
\hline 1 & ppos & $\begin{array}{l}\text { The proportion of Pareto-optimal solu- } \\
\text { tions measures the size of the Pareto } \\
\text { front relative to the population size. A } \\
\text { high and especially strongly increasing } \\
\text { ppos may indicate a problem of domi- } \\
\text { nance resistance. } \\
\text { The hypervolume is the volume in the } \\
\text { objective space covered by the Pareto- } \\
\text { optimal solutions. An increasing } h v \text { in- } \\
\text { dicates that the search is able to find im- } \\
\text { proved solutions, otherwise the } h v \text { and } \\
\text { search stagnate. }\end{array}$ & $\begin{array}{l}\text { A drastically increasing ppos has been } \\
\text { observed for } 2 / 5 \text { apps and to a smaller } \\
\text { extent for further } 2 / 5 \text { apps indicating } \\
\text { that the search of SAPIENZ could suffer } \\
\text { from dominance resistance. However, } \\
\text { for } 1 / 5 \text { apps ppos remained low. } \\
\text { The } h v \text { stagnates for } 4 / 5 \text { apps after } 25 \\
\text { and for } 1 / 5 \text { apps already after five gen- } \\
\text { erations indicating a stagnation of the } \\
\text { search in SAPIENZ, potentially relatively } \\
\text { early in the search process. }\end{array}$ \\
\hline \multicolumn{4}{|c|}{ Metrics for the diversity of the population } \\
\hline 3 & maxdiam & $\begin{array}{l}\text { The maximum population diameter quan- } \\
\text { tifies the largest distance between any } \\
\text { two individuals and thus the maximum } \\
\text { spread of the population in the search } \\
\text { space. }\end{array}$ & $\begin{array}{l}\text { During the first } 25 \text { generations, maxdiam } \\
\text { decreases for all apps, in } 3 / 5 \text { apps even } \\
\text { drastically, denoting a decrease of the } \\
\text { maximum spread of the population } \\
\text { in the search space. }\end{array}$ \\
\hline 4 & avgdiam & $\begin{array}{l}\text { The average population diameter is the } \\
\text { average of all pairwise distances between } \\
\text { all individuals, which quantifies the av- } \\
\text { erage diversity of the population. }\end{array}$ & $\begin{array}{l}\text { During the first } 25 \text { generations, avgdiam } \\
\text { decreases for } 5 / 5 \text { apps drastically denot- } \\
\text { ing a decrease of the average popu- } \\
\text { lation diversity. }\end{array}$ \\
\hline 5 & mindiam & $\begin{array}{l}\text { The minimum population diameter quan- } \\
\text { tifies the smallest distance between any } \\
\text { two individuals and thus the minimum } \\
\text { diversity of the population. }\end{array}$ & $\begin{array}{l}\text { The mindiam decreases for } 5 / 5 \text { apps } \\
\text { quickly reaching a value of } 0 \text {, which de- } \\
\text { notes that the population contains du- } \\
\text { plicate solutions. }\end{array}$ \\
\hline 6 & reldiam & $\begin{array}{l}\text { The relative population diameter is avg- } \\
\text { diam divided by the largest possible dis- } \\
\text { tance between two individuals. It indi- } \\
\text { cates the concentration of the popula- } \\
\text { tion in the search space. }\end{array}$ & $\begin{array}{l}\text { During the first } 25 \text { generations, reldiam } \\
\text { decreases for } 5 / 5 \text { apps indicating a con- } \\
\text { centration/grouping of all individ- } \\
\text { uals of the population in the search } \\
\text { space. }\end{array}$ \\
\hline \multicolumn{4}{|c|}{ Metrics for the diversity of the Pareto-optimal solutions } \\
\hline 7 & pconnec & $\begin{array}{l}\text { The proportion of Pareto-optimal solu- } \\
\text { tions in clusters indicates the degree of } \\
\text { grouping (clustering) of Pareto-optimal } \\
\text { solutions in the search space. }\end{array}$ & $\begin{array}{l}\text { pconnec increases so that for } 2 / 5 \text { apps } \\
\text { most and for } 3 / 5 \text { apps even all Pareto- } \\
\text { optimal solutions are part of clus- } \\
\text { ters in the search space. }\end{array}$ \\
\hline 8 & nconnec & $\begin{array}{l}\text { The number of clusters counts in how } \\
\text { many regions of the search space the } \\
\text { Pareto-optimal solutions are clustered. }\end{array}$ & $\begin{array}{l}\text { For } 3 / 5 \text { apps, there exists one cluster } \\
\text { containing all Pareto-optimal solu- } \\
\text { tions except of } 2 / 5 \text { apps with either one } \\
\text { or two, or more than three clusters. }\end{array}$ \\
\hline 9 & kconnec & $\begin{array}{l}\text { The minimum distance } k \text { for a connected } \\
\text { graph determines the maximum spread } \\
\text { of all Pareto-optimal solutions in the } \\
\text { search space. }\end{array}$ & $\begin{array}{l}\text { kconnec decreases drastically for } 3 / 5 \text { and } \\
\text { moderately for } 2 / 5 \text { apps, denoting a de- } \\
\text { creasing spread of Pareto-optimal } \\
\text { solutions in the search space. }\end{array}$ \\
\hline 10 & lconnec & $\begin{array}{l}\text { The number of Pareto-optimal solutions } \\
\text { in the largest cluster quantifies the abso- } \\
\text { lute size of the largest cluster and thus, } \\
\text { how many Pareto-optimal solutions are } \\
\text { located in the most dense area of the } \\
\text { search space. }\end{array}$ & $\begin{array}{l}\text { lconnec increases for } 3 / 5 \text { apps denot- } \\
\text { ing that the largest cluster contains } \\
\text { many }(20-50) \text { Pareto-optimal solu- } \\
\text { tions, whereas for } 2 / 5 \text { apps the largest } \\
\text { cluster contains }<10 \text { solutions given a } \\
\text { smaller ppos. }\end{array}$ \\
\hline 11 & hvconnec & $\begin{array}{l}\text { The proportion of hypervolume covered by } \\
\text { the largest cluster quantifies the size of } \\
\text { the largest cluster in the objective space, } \\
\text { that is, how much the most dense area of } \\
\text { the search space contributes to the over- } \\
\text { all hypervolume. }\end{array}$ & $\begin{array}{l}\text { For } 5 / 5 \text { apps, hvconnec converges to } \\
\text { a high value so that the overall } \\
\text { hypervolume is mainly (2/5) or } \\
\text { completely (3/5) achieved by the } \\
\text { largest cluster making the remaining } \\
\text { clusters less relevant in terms of fitness. }\end{array}$ \\
\hline
\end{tabular}


concentrated in the search space ( $c f .[20])$. Consequently, the diversity of the whole population decreases during the first 25 generations, after which SAPIENZ loses its ability to produce fitter test suites (evolvability).

Thirdly, we investigated how the diversity of the Pareto-optimal solutions develops as the diversity of the whole population decreases. The development of the proportion of Pareto-optimal solutions in clusters (pconnec) indicates a grouping of these solutions in one or more areas of the search space. Considering the number of clusters (nconnec), these solutions are often grouped in one or very few clusters and thus, they are located in one or very few areas of the search space. Regardless of the actual number of clusters, the decreasing minimum distance $k$ required to form one cluster of all Pareto-optimal solutions (kconnec) shows a declining spread of all Pareto-optimal solutions in the search space. Moreover, considering the largest actual cluster, we notice that this cluster is often indeed large in terms of number of Pareto-optimal solutions (lconnec), and hypervolume covered by these solutions (hvconnec). Even if there exist multiple clusters of Pareto-optimal solutions, the largest one still contributes most to the overall hypervolume and thus, to the final Pareto front of the search. This indicates a grouping process of the Pareto-optimal solutions toward one (large) cluster in the search space that dominates in the objective space. Hence, the most-promising test suites are found by SAPIEnz in one area of the search space. Consequently, we observe a decreasing diversity of the Pareto-optimal solutions during the search.

In summary, the fitness landscape analysis of SAPIENZ conducted for the selected five apps indicates a stagnation of the search (declining evolvability) while the diversity of all solutions decreases in the search space. Thus, the loss of diversity affects the dominated and non-dominated solutions.

\section{SAPIENZ ${ }^{d i v}$}

Given the fitness landscape analysis results summarized in Section 3.4, SAPIENZ suffers from a decreasing diversity of solutions in the search space over time. It is known that the performance of genetic algorithms is influenced by diversity $[10,14,25]$. A low diversity may lead the search to a local optimum that cannot be escaped easily $[10,25]$. Thus, diversity is important to address dominance resistance so that the search can produce new solutions that dominate poorly performing, locally non-dominated solutions [17]. Moreover, Shir et al. [26, p. 95] report that promoting diversity in the search space does not hamper "the convergence to a precise and diverse Pareto 
front approximation in the objective space of the original algorithm". This statement suggests that promoting diversity in SAPIENZ will not degrade the performance of SAPIENZ but might rather achieve improvements.

Therefore, we extended SAPIENZ to SAPIEnz ${ }^{\text {div }}$ by integrating mechanisms into the search algorithm that promote the diversity of the solutions being evolved in the search space. ${ }^{3}$ We developed four mechanisms that extend the SAPIENZ algorithm at different steps: at the initialization, before and after the variation, and at the selection. Algorithm 2 shows the extended search algorithm of SAPIENZ ${ }^{d i v}$ and highlights the novel mechanisms in blue. In the following, we discuss these mechanisms.

Diverse initial population. Considering Algorithm 2, initializing SAPIENZ comprises the following steps: Initializing the UI model, Pareto front, test reports, and the counter for the generations (lines 1 and 2). Booting up the Android devices or emulators to be used for executing the app under test (line 3). Installing the Motifcore component of SAPIEnz, responsible for the exploration and evaluation of tests, to the devices or emulators (line 4). Statically analyzing the app under test for seeding realistic Strings to be used by test to fill text fields in the app (line 5). ${ }^{4}$ Instrumenting (for measuring coverage) and installing the app under test to the devices or emulators.

Afterwards, SAPIENZ creates the initial population. Since the initial population may affect the results of the search [27], we assume that a diverse initial population could be a better start for the exploration. Thus, we extend the generation of the initial population $P_{\text {init }}$ to promote diversity. Instead of generating $|P|=$ size $_{\text {pop }}$ solutions, we generate size $_{\text {init }}$ solutions where size $_{\text {init }}>$ size $_{\text {pop }}$ (line 7 in Algorithm 2). Then, we select those size pop $_{\text {so- }}$ lutions from $P_{\text {init }}$ that are most distant from each other using Algorithm 1, to form the first population $P$ (line 8). Subsequently, $P$ is evaluated by computing the fitness of its members (line 9).

Adaptive diversity control. This mechanism dynamically controls the diversity if the population members are becoming too close in the search space relative to the initial population. It further makes the SAPIENZ algorithm adaptive as it uses feedback of the search to adapt the search (cf. [10]).

\footnotetext{
${ }^{3}$ SAPIENZ ${ }^{d i v}$ is available at: https://github.com/thomas-vogel/sapienzdiv-ssbse19.

${ }^{4}$ String seeding as well as the use of the so called motif genes in SAPIENz have been deactivated in the scope of this work similar to the evaluation of SAPIENZ in [1].
} 


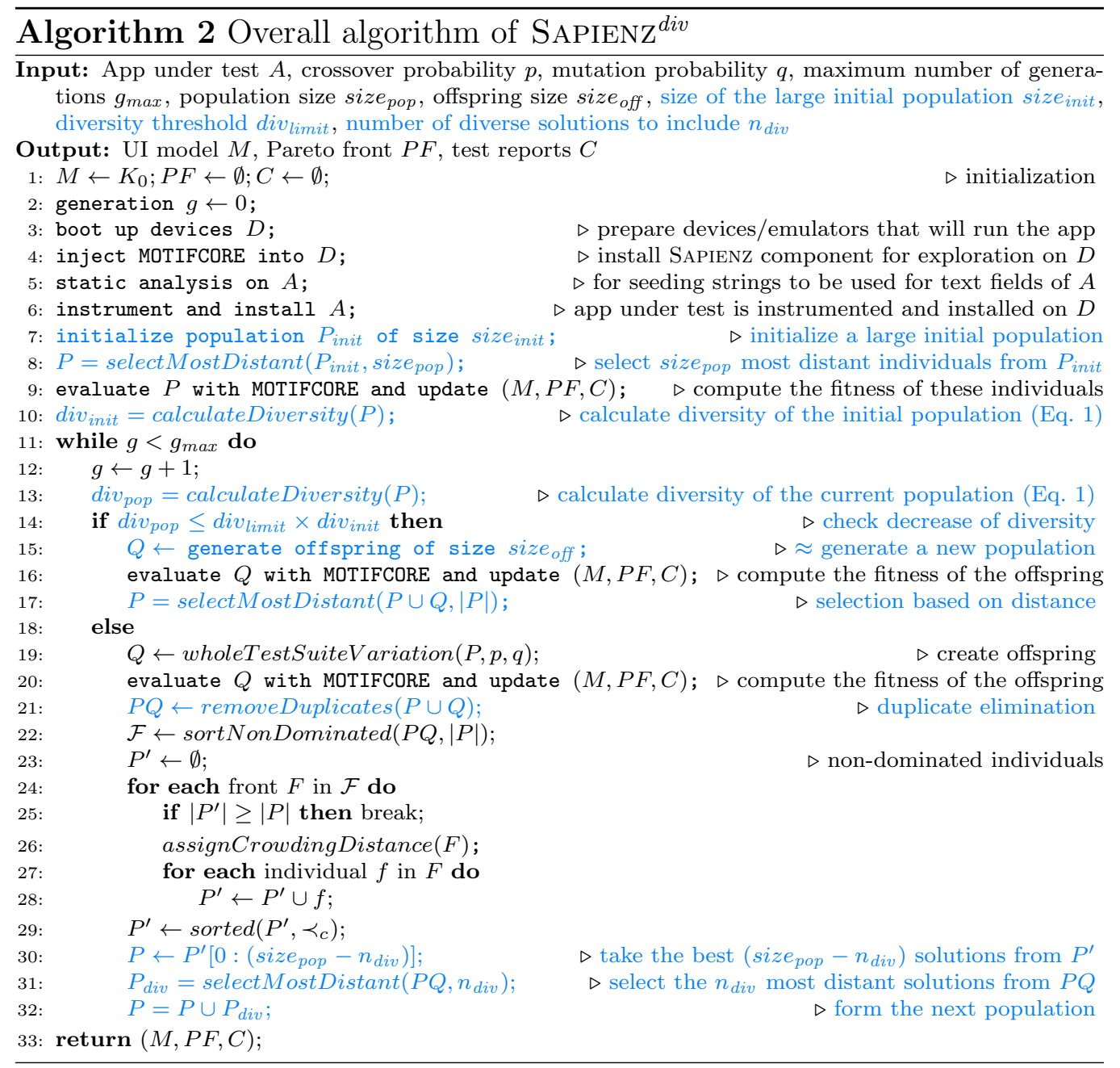

To quantify the diversity $d_{i v}$ pop of population $P$, we use the average population diameter (avgdiam) defined in Eq. 1 (Section 3.3.2). At the beginning of each generation, $d i v_{\text {pop }}$ of the current population $P$ is calculated (line 13) and compared to the diversity of the initial population div $_{\text {init }}$ calculated once in line 10. The comparison in line 14 checks whether div $v_{p o p}$ has decreased to less than div $_{\text {limit }} \times d i v_{\text {init }}$. For example, the condition is satisfied for the given threshold div $_{\text {limit }}=0.4$ if the diversity of the current population ( div $\left._{\text {pop }}\right)$ has decreased to less than $40 \%$ of the diversity of the initial population (div init $_{\text {) }}$ ).

In this case, the offspring $Q$ is obtained by generating new solutions using the original SAPIENZ method to initialize a population (line 15). After 
evaluating the fitness of the offspring (line 16), the next population is formed by selecting the $|P|$ most distant individuals from the current population $P$ and offspring $Q$ (line 17). In the other case, when the diversity of $P$ is at an acceptable level, the variation operators (crossover and mutation) of SAPIENZ are applied to obtain the offspring (line 19). After evaluating the fitness of the offspring (line 20), the selection based on NSGA-II is applied (lines 2229). Thus, this mechanism promotes diversity on demand by inserting new individuals to the population, having an effect of restarting the search.

Duplicate elimination. The fitness landscape analysis found duplicated test suites in the population. Eliminating duplicates is one technique to maintain diversity and improve search performance $[10,28,29]$. Thus, we remove duplicates after reproduction and before selection in the current population and offspring (line 21). Duplicated test suites are identified by a distance of 0 computed by our distance metric $\operatorname{dist}\left(t_{1}, t_{2}\right)$ defined in Algorithm 1.

Hybrid selection. This mechanism extends the selection in SAPIENZ to promote diversity in the search space. For this purpose, the selection is divided in two parts: (1) The non-dominated sorting of NSGA-II is performed as in SAPIENZ (lines 22-29 in Algorithm 2) to obtain the solutions $P^{\prime}$ sorted by domination rank and crowding distance. (2) From $P^{\prime}$, the best $\left(s i z e_{p o p}-n_{\text {div }}\right)$ solutions form the next population $P$ where size $e_{p o p}$ is the size of $P$ and $n_{\text {div }}$ the configurable number of diverse solutions to be included in $P$ (line 30). These $n_{d i v}$ diverse solutions $P_{d i v}$ are selected as the most distant solutions from the current population and offspring $P Q$ (line 31) using our distance metric dist $\left(t_{1}, t_{2}\right)$ defined in Algorithm 1. Finally, $P_{\text {div }}$ is added to the next population $P$ (line 32 ). After selection, the next evolutionary epoch begins.

While the NSGA-II sorting considers the diversity of solutions in the objective space (crowding distance), the selection of SAPIENZ ${ }^{\text {div }}$ also considers the diversity of solutions in the search space. Thus, the selection of SAPIENZ $^{\text {div }}$ is hybrid taking the objective and search space into account.

\section{Evaluation}

We evaluate SAPIEnz ${ }^{\text {div }}$ in a head-to-head comparison with SAPIENz to investigate the benefits of the diversity-promoting mechanisms. We consider SAPIENZ as the baseline for our approach since Mao et al. [1] have already shown that SAPIENZ significantly outperforms Monkey (random testing) [30] and Dynodroid [31]. Thus, we do not compare SAPIENZ ${ }^{\text {div }}$ against random 
testing. We also do not compare SAPIENZ ${ }^{\text {div }}$ against other app testing approaches (e.g., Dynodroid) since we want to evaluate whether our adaptation of SAPIENZ informed by the fitness landscape analysis of SAPIENZ achieves any improvements over the baseline being naturally SAPIENZ. In theory, we could have selected any search-based app testing approach to analyze its fitness landscape and adapt accordingly its heuristic, in which case the selected approach would be the baseline. We selected SAPIENZ as it was the state of the art at the time when we have started this work. Thus, our evaluation is a head-to-head comparison between SAPIEnz ${ }^{d i v}$ and SAPIENZ targeting the following four research questions (RQ):

RQ1 How does the runtime overhead of SAPIENZ ${ }^{\text {div }}$ compare to SAPIENZ?

RQ2 How does the coverage achieved by SAPIENZ ${ }^{\text {div }}$ compare to SAPIENZ? RQ3 How do the faults found by SAPIEnZ ${ }^{\text {div }}$ compare to SAPIENZ?

RQ4 How does SAPIEnz ${ }^{\text {div }}$ compare to SAPIENZ concerning the length of their fault-revealing test sequences?

Research questions similar to RQ2, RQ3, and RQ4 have been investigated by Mao et al. [1] to evaluate SAPIEnz. Since SAPIEnz div adds diversitypromoting mechanisms to SAPIENZ, it adds a runtime overhead to SAPIENZ that we investigate with RQ1. To answer all RQs, we conduct a comprehensive empirical study that we discuss in the following.

\subsection{Novelty With Respect to Our Previous Study}

The comprehensive study presented in this article extends our study from previous work [7]. Our previous study was preliminary since we run each SAPIENZ and SAPIENZ ${ }^{\text {div }} 20$ times on 10 apps while limiting the search to 10 generations because of the high execution costs of SAPIENZ and SAPIENZ ${ }^{d i v}$. Still, the experiment for the previous study required in total almost 32 days of computation time (measured wall-clock time of the whole experiment). For this article, we conduct a new comprehensive study by executing each SAPIENZ and SAPIENZ ${ }^{\text {div }} 30$ times on 34 apps while performing the search over 40 generations. The new experiment for the comprehensive study required in total 562 days of computation time (Table 4).

For the new study, we selected the 34 apps follows. We selected nine of the ten $\operatorname{apps}^{5}$ used to statistically evaluate SAPIENZ [1]. These apps have

\footnotetext{
${ }^{5}$ We excluded Droidsat as this app requests information from a website whose owner
} 
Table 4: Overview of the new study extending our previous study [7].

\begin{tabular}{lrr}
\hline & Previous study [7] & New study \\
\hline \# Apps & 10 & 34 \\
\# Generations & 10 & 40 \\
\# Repetitions & 20 & 30 \\
Total computation time (days) & 32 & 562 \\
\hline
\end{tabular}

also been used in our preliminary study. As novel apps, we further selected 21 apps from Su et al. [32] and we choose randomly four apps from F-Droid.

Besides testing more apps with more repetitions, the major difference between the previous and new study is the number of generations that an approach searches for test suites for an app. Extending the number of generations from 10 to 40 is motivated by the results of the fitness landscape analysis ( $c f$. Section 3) showing that the search of SAPIENZ stagnates after 25 generations. Thus, this extension allows us to investigate whether the diversity-promoting mechanisms introduced in SAPIENZ ${ }^{\text {div }}$ will have an effect on the performance after the search of SAPIENZ actually stagnates.

\subsection{Experimental Setup and Research Protocol}

To answer the four research questions, we conducted an empirical study, in which we compare the two approaches SAPIENZ and SAPIENZ ${ }^{\text {div }}$ by measuring and analyzing their performance in terms of execution time, achieved coverage, triggered faults, and length of the fault-revealing test cases. Thus, the independent variable is the testing approach and it has two levels: SAPIENZ and SAPIEnZ ${ }^{\text {div }}$. The dependent variables are the execution times, achieved coverage, triggered faults, and length of fault-revealing test cases. For this purpose, we execute each approach on each of the 34 subjects (apps) whose selection was discussed in the previous section. For each approach and app, we observe and analyze the performance of the approach after 40 generations of search. ${ }^{6}$ To enable a statistical analysis, we repeat the execution for each app and each approach 30 times. The execution of the experiment

complained about the traffic caused by the tests generated and executed by SAPIENZ and SAPIENZ $^{\text {div }}$ while conducting the experiments.

${ }^{6}$ To take the overhead of SAPIENZ ${ }^{d i v}$ into account, we will later reduce the number of generations for SAPIENZ ${ }^{d i v}$ to enable a fair comparison with SAPIENZ concerning the achieved coverage, triggered faults, and the length of fault-revealing test cases. 
was distributed on eight servers ${ }^{7}$ where each server runs one approach to test one app at a time using ten Android emulators in parallel (Android KitKat, API 19).

For each concern (i.e., execution time, achieved coverage, triggered faults, and length of the fault-revealing test cases), we compare SAPIENZ and SAPIENZ ${ }^{\text {div }}$ for each individual app. Thus, we compare 30 samples of SAPIENZ with 30 samples of SAPIENZ ${ }^{\text {div }}$, whereas one sample is obtained by one run of an approach on the app. The sample sets between SAPIENz and SAPIEnz ${ }^{\text {div }}$ are consequently independent. Considering such independent samples and since we cannot make any assumption about the distribution of the results, we perform a non-parametric Mann-Whitney U test [33, 34, 35] for each concern to check whether the measured values of the concern achieved by both approaches differ significantly for each subject with a $95 \%$ confidence level $(p<0.05)$. Moreover, we use the Vargha-Delaney effect size $\hat{A}_{12}[36]$ to measure the probability that running SAPIENZ ${ }^{\text {div }}$ yields better concern values than running SAPIENZ ( $c f$. [35]). We further characterize small, medium, and large differences between SAPIENZ ${ }^{d i v}$ and SAPIENZ with respect to a concern if $\hat{A}_{12}>0.56, \hat{A}_{12}>0.64$, and $\hat{A}_{12}>0.71$ respectively. These thresholds have been proposed by Vargha and Delaney [36] and used by Mao et al. [1] in the original evaluation of SAPIENZ.

To give SAPIEnZ and SAPIEnz ${ }^{\text {div }}$ the same conditions, both use the default SAPIENZ configuration [1] that has been also used for the fitness landscape analysis ( $c f$. Table 2 in Section 3.2). Moreover, we configured the novel parameters of SAPIENZ ${ }^{\text {div }}$ as follows (Table 5): The size of the large initial population $\left(\right.$ size $\left._{\text {init }}\right)$ is 100 , the diversity threshold $\left(\right.$ div $\left._{\text {limit }}\right)$ is 0.5 , and the number of diverse solutions to be included in the selection phase $\left(n_{\text {div }}\right)$ is 15 . We have been conservative in setting these parameters without any parameter tuning. size init $_{\text {in }}$ is just twice the size of the population $(|P|=50)$, div limit $_{\text {it }}$ requires that the diversity of a population must decrease by at least $50 \%$ compared to the diversity of the initial population to trigger the adaptive diversity control mechanism, and $n_{\text {div }}$ determines that just 15 individuals out of the 50 population members will be selected based on diversity rather than fitness. A less conservative parameter setting would use larger values for size $e_{\text {init }}$ and $n_{\text {div }}$ and a lower value for div $v_{\text {limit }}$.

\footnotetext{
${ }^{7}$ For each server: $2 \times \operatorname{Intel}(\mathrm{R})$ Xeon(R) CPU E5-2620 @ 2.00GHz, with 64GB RAM.
} 
Table 5: SAPIEnZ ${ }^{d i v}$ configuration.

\begin{tabular}{lr}
\hline Parameter & Value \\
\hline Size of the large initial population $($ size init $)$ & 100 \\
Diversity threshold $\left(\right.$ div $\left._{\text {limit }}\right)$ & 0.50 \\
Number of diverse solutions included in the selection phase $\left(n_{\text {div }}\right)$ & 15 \\
\hline
\end{tabular}

\subsection{Results}

In the following, we present the results of the empirical study by answering each of the four research questions.

\subsubsection{Execution Time (RQ1)}

To determine the runtime overhead of SAPIEnz ${ }^{\text {div }}$, we measured the execution time of each approach (SAPIENZ and SAPIENZ ${ }^{\text {div }}$ ) to test an app over 40 generations of search. The corresponding results for each of the 34 apps are shown Table 6. For each app the table lists (i) the version of the app, (ii) the mean, median, and standard deviation (SD) of the execution times of the 30 runs of SAPIENZ as well as SAPIENZ ${ }^{\text {div }}$, (iii) the p-value (printed bold if $p<0.05)$ obtained by the Mann-Whitney $\mathrm{U}$ test checking significant differences between the SAPIENZ and SAPIEnZ ${ }^{d i v}$ samples, (iv) the $\hat{A}_{12}$ effect size comparing SAPIENZ ${ }^{\text {div }}$ to SAPIENZ $^{8}$, and $(\mathrm{v})$ the percentagewise overhead of SAPIENZ $^{\text {div }}$ with respect to SAPIENZ based on the median execution times.

Moreover, each $\hat{A}_{12}$ value is followed by a triangle. This triangle points upwards $(\boldsymbol{\Delta} / \triangle)$ if SAPIENZ ${ }^{\text {div }}$ outperforms SAPIEnZ, downwards $(\mathbf{\nabla} / \nabla)$ if SAPIEnz Siv $^{\text {S }}$ is outperformed by SAPIENZ, and to the right $(\nabla / \triangleright)$ if SAPIENZ ${ }^{d i v}$ performs equally to SAPIENZ. A triangle is further filled black if the corresponding results are statistically significant based on the Mann-Whitney U test with a $95 \%$ confidence level. Otherwise, the triangles are filled white.

Concerning the results of Table 6, SAPIEnz significantly outperforms SAPIEnZ $^{\text {div }}$ with large effect size on $34 / 34$ apps for execution time. ${ }^{8}$ From a practical perspective, the runtime overhead of SAPIENZ ${ }^{\text {div }}$ with respect to SAPIEnZ ranges from $5.47 \%$ for the app Yaab up to $46.57 \%$ for the app Kindmind. In absolute terms, for the Yaab app the median execution times

\footnotetext{
${ }^{8}$ When comparing both approaches from the perspective of SAPIENZ, i.e., when switching the comparison from "SAPIEnz div vs. SAPIEnz" to "SAPIEnz vs. SAPIEnz" div", the effect size values must be inverted ( 1 - effect size value).
} 
Table 6: Execution time (minutes) of both approaches running over 40 generations on each of the 34 apps.

\begin{tabular}{|c|c|c|c|c|c|c|c|c|c|c|}
\hline Subject & Version & Mean & $\begin{array}{l}\text { SAPIENZ } \\
\text { Median }\end{array}$ & $\mathrm{SD}$ & Mean & $\begin{array}{l}\text { SAPIENZ }^{d i} \\
\text { Median }\end{array}$ & $\mathrm{SD}$ & p-value & $\hat{A}_{12}$ & Overhead \\
\hline BabyCare & 1.5 & 372.00 & 368.70 & 27.66 & 470.02 & 470.71 & 57.99 & 0.000000 & $0.03 \boldsymbol{\nabla}$ & $27.67 \%$ \\
\hline Arity & & 268.95 & 262.98 & 26.82 & 295.09 & 296.94 & 15.33 & 0.000001 & $0.13 \boldsymbol{\nabla}$ & $12.92 \%$ \\
\hline JustSit & .3 .3 & 295.57 & 290.89 & 31.58 & 331.02 & 329.90 & 17.89 & 0.000000 & $0.09 \boldsymbol{\nabla}$ & $13.41 \%$ \\
\hline Hydrate & 1.5 & 302.19 & 299.17 & 11.42 & 347.42 & 347.95 & 13.25 & 0.000000 & $0.01 \nabla$ & $16.31 \%$ \\
\hline FillUp & 1.7 .2 & 364.09 & 363.38 & 24.61 & 455.44 & 451.96 & 39.75 & 0.000000 & $0.02 \mathbf{\nabla}$ & $24.38 \%$ \\
\hline Kanji & 1.0 & 374.08 & 366.56 & 30.38 & 465.75 & 451.12 & 50.88 & 0.000000 & $0.05 \boldsymbol{\nabla}$ & $23.07 \%$ \\
\hline BookWorm & 1.0 .18 & 332.84 & 327.43 & 27.41 & 387.77 & 386.44 & 24.11 & 0.000000 & $0.05 \boldsymbol{\nabla}$ & $18.02 \%$ \\
\hline Maniana & 1.26 & 422.60 & 409.19 & 51.25 & 507.08 & 498.24 & 33.01 & 0.000000 & $0.06 \boldsymbol{\nabla}$ & $21.76 \%$ \\
\hline L9Droid & 0.6 & 322.38 & 326.44 & 27.23 & 406.66 & 401.75 & 31.04 & 0.000000 & $0.01 \nabla$ & $23.07 \%$ \\
\hline Yaab & 1.10 .1 & 379.84 & 376.92 & 38.37 & 413.00 & 397.53 & 49.94 & 0.002016 & & $5.47 \%$ \\
\hline Budget & 4.2 & 398.11 & 397.91 & 23.11 & 468.45 & 467.57 & 27.96 & & $.02 \mathbf{\nabla}$ & $17.51 \%$ \\
\hline Campyre & 1.0 .1 & 317.49 & 315.50 & 25.08 & 397.75 & 386.07 & 33.01 & & $0.02 \boldsymbol{\nabla}$ & $22.37 \%$ \\
\hline URLazy & $1.0 \mathrm{a}$ & 208.37 & 209.20 & 6.19 & 253.04 & 249.98 & 14.53 & 0.000000 & $0.00 \mathbf{\nabla}$ & $19.49 \%$ \\
\hline ArXiv & 2.0 .22 & 377.52 & 383.23 & 52.85 & 475.32 & 475.04 & 58.22 & 0.000000 & $0.11 \boldsymbol{\nabla}$ & $23.96 \%$ \\
\hline Cetoolbox & 1.0 & 308.14 & 309.46 & 24.19 & 399.87 & 400.57 & 17.96 & 0.000000 & $0.00 \boldsymbol{\nabla}$ & $29.44 \%$ \\
\hline CurrencyConverter & 1.1 & 343.27 & 346.02 & 28.56 & 433.88 & 433.99 & 23.17 & 0.000000 & $0.01 \mathrm{\nabla}$ & $25.42 \%$ \\
\hline Charmap & 1.0 .1 & 479.74 & 456.31 & 94.72 & 627.55 & 615.12 & 62.36 & 0.000000 & $0.10 \boldsymbol{\nabla}$ & $34.80 \%$ \\
\hline Nan & 0.8 .98 & 353.04 & & 29.39 & 445. & 431.29 & 48.41 & & & $23.20 \%$ \\
\hline Ana & 1.0 & 286.95 & & 31.25 & 354. & 373.74 & 45.77 & & & $56 \%$ \\
\hline Kindmind & 1.0.0_BETA & 526.35 & & 80.69 & & 724.07 & 158.94 & & & $57 \%$ \\
\hline Urforms & 1.12 & 446.86 & 444.78 & 37.45 & 556.92 & 538.33 & 65.36 & 0.000000 & $4 \mathbf{v}$ & $21.03 \%$ \\
\hline manager & 1.0.1.8 & 338.18 & 339.40 & 18.65 & 386.95 & 385.65 & 20.16 & 0.000000 & $0.04 \boldsymbol{\nabla}$ & $13.63 \%$ \\
\hline Remembeer & 1.3 .0 & 328.46 & 334.85 & 20.15 & 429.67 & 416.05 & 52.30 & 0.000000 & $0.00 \boldsymbol{\nabla}$ & $24.25 \%$ \\
\hline Pockettalk & 2.5 & 223.61 & 223.73 & 10.17 & 283.32 & 283.25 & 8.34 & 0.000000 & $0.00 \boldsymbol{\nabla}$ & $26.60 \%$ \\
\hline Rot13 & 1.0 .2 & 331.86 & 332.27 & 24.71 & 415.20 & 410.61 & 15.56 & 0.000000 & $0.00 \mathbf{\nabla}$ & $23.58 \%$ \\
\hline IShows & 6.2 & 275.80 & 263.44 & 36.31 & 354. & 319.93 & 72.96 & & $0.14 \mathbf{\nabla}$ & $21.44 \%$ \\
\hline Episodes & 0.7 & 277.31 & 274.20 & 22.68 & 380.89 & 380.97 & 34.36 & 0.000000 & $0.00 \boldsymbol{\nabla}$ & $38.94 \%$ \\
\hline Angulo & 2.0 & 274.24 & 272.82 & 13.37 & 315.58 & 314.98 & 12.30 & 0.000000 & $0.00 \mathrm{\nabla}$ & $15.46 \%$ \\
\hline Rightsalert & $0.3 \mathrm{a}$ & 761.54 & 754.36 & 96.06 & 838.66 & 852.32 & 69.20 & 0.000346 & $0.24 \mathbf{\nabla}$ & $12.99 \%$ \\
\hline ApkTrack & $1.1 \mathrm{~h}$ & 388.90 & 382.21 & 38.73 & 471.67 & 442.89 & 73.74 & 0.000000 & $0.06 \boldsymbol{\nabla}$ & $15.88 \%$ \\
\hline Diary & 1.0 & 264.54 & 262.43 & 12.25 & 314.07 & 313.08 & 22.57 & 0.000000 & $0.02 \boldsymbol{\nabla}$ & $19.30 \%$ \\
\hline & 2.2 & 293.49 & 288.48 & 20.38 & 385.84 & 373.14 & 72.16 & 0.000000 & $0.01 \mathrm{\nabla}$ & $29.35 \%$ \\
\hline Fakedawn & 1.3 & 326.72 & 322.24 & 17.18 & 434.45 & 421.64 & 49.38 & 0.000000 & & $30.84 \%$ \\
\hline Klaxon & 0.27 & 250.91 & 251.29 & 9.66 & 292.45 & 292.43 & 9.94 & 0.000000 & $0.00 \boldsymbol{\nabla}$ & $16.37 \%$ \\
\hline
\end{tabular}

is 376.92 minutes (6.28 hours) for SAPIENZ and 397.53 minutes (6.63 hours) for SAPIENZ ${ }^{\text {div }}$ resulting in an overhead of 20.61 minutes for a search over 40 generations. In contrast, Kindmind exemplifies a drastic overhead of SAPIENZ $^{d i v}$. In this case, the median execution times is 494.01 minutes (8.23 hours) for SAPIENZ and 724.07 minutes (12.07 hours) for SAPIENZ ${ }^{\text {div }}$ resulting in an absolute overhead of 230.06 minutes (3.83 hours) for a search over 40 generations. Thus, the diversity-promoting mechanisms of SAPIENz ${ }^{\text {div }}$ add a significant overhead to SAPIENZ. 
Answer to RQ1: Based on our evaluation, we conclude that SAPIENZ ${ }^{\text {div }}$ adds a significant runtime overhead to SAPIENZ that was observed for $34 / 34$ apps and that ranges from $5.47 \%$ to $46.57 \%$ for individual apps. In our experiments, this resulted in an absolute overhead of SAPIENZ ${ }^{\text {div }}$ ranging from around 21 minutes to 3.8 hours compared to SAPIENZ when generating test suites for an individual app over 40 generations.

\subsubsection{Coverage (RQ2)}

To analyze the coverage achieved by SAPIENz ${ }^{d i v}$ and SAPIENZ, we measured the final percentagewise statement coverage achieved at the end of the search by each approach. To make a fair coverage-based comparison between SAPIENZ $^{d i v}$ and SAPIENZ, we have to take the overhead of SAPIENZ ${ }^{d i v}$ into account ( $c f$. Section 5.3.1). Consequently, for each app we consider the coverage results of SAPIENZ achieved after 40 generations of search, whereas we reduce the number of generations of search for SAPIENZ ${ }^{d i v}$ according to the app-specific runtime overhead of SAPIENZ ${ }^{d i v}$. For instance, SAPIENZ ${ }^{d i v}$ has a runtime overhead of $27.67 \%$ for the app BabyCare (Table 6) so that we reduce the number of generations by the same fraction $(40 \times 27.67 \%=11.07)$. Thus, we consider the coverage results of SAPIENZ ${ }^{d i v}$ for BabyCare after 29 generations of search. Similarly, we compute for all apps the numbers of generations considered for SAPIENZ ${ }^{d i v}$ to enable a fair comparison.

The corresponding coverage results of our study is shown in Table 7. Similarly to Table 6 , this table lists for each app the mean, median, and standard deviation (SD) of the coverage results for SAPIENZ and SAPIENZ ${ }^{d i v}$, the pvalue obtained by the Mann-Whitney $U$ test checking significant differences between the SAPIENZ and SAPIENZ div coverage results, and $\hat{A}_{12}$ effect size comparing SAPIEnZ ${ }^{d i v}$ to SAPIEnZ ${ }^{8}$. Moreover, the table lists the number of generations after which we consider the coverage results for the comparisonbeing always 40 for SAPIENZ and fewer generations for SAPIENZ ${ }^{d i v}$.

Considering the results of Table 7 , we obtained statistically significant results for $9 / 34$ apps. For all of these nine apps, SAPIENZ ${ }^{\text {div }}$ significantly outperforms SAPIENZ with respect to coverage, in 3/9 cases with large effect size, in $3 / 9$ cases with medium effect size, and in $3 / 9$ cases with small effect size. In contrast, SAPIENZ ${ }^{d i v}$ was never significantly outperformed by SAPIEnz. Concerning the statistically significant results for the nine apps from a practical perspective, SAPIEnZ ${ }^{d i v}$ was able to increase the median 
Table 7: Final statement coverage (\%) achieved by SAPIENZ after 40 generations (Gen.) and by SAPIENZ ${ }^{d i v}$ after fewer generations (Gen.) of search taking the app-specific overhead of SAPIENZ ${ }^{d i v}$ into account.

\begin{tabular}{|c|c|c|c|c|c|c|c|c|c|c|}
\hline Subject & Gen. & $\begin{array}{c}\text { SAF } \\
\text { Mean }\end{array}$ & $\begin{array}{l}\text { IENZ } \\
\text { Median }\end{array}$ & SD & \multicolumn{3}{|c|}{ SAPIENZ ${ }^{d i v}$} & SD & p-value & $\hat{A}_{12}$ \\
\hline BabyCare & 40 & 39.00 & 39.00 & 1.64 & 29 & 38.73 & 39.00 & 1.05 & 0.361919 & $0.47 \nabla$ \\
\hline Arity & 40 & 77.53 & 78.00 & 1.33 & 35 & 78.00 & 78.00 & 1.02 & 0.065616 & $0.61 \Delta$ \\
\hline JustSit & 40 & 64.53 & 64.00 & 1.36 & 35 & 64.93 & 65.00 & 1.80 & 0.237130 & $0.55 \Delta$ \\
\hline Hydrate & 40 & 47.47 & 48.00 & 0.90 & 33 & 47.87 & 48.00 & 1.11 & 0.073156 & $0.60 \Delta$ \\
\hline FillUp & 40 & 44.70 & 44.50 & 2.41 & 30 & 44.03 & 44.00 & 2.70 & 0.203496 & $0.44 \nabla$ \\
\hline Kanji & 40 & 66.47 & 66.00 & 0.51 & 31 & 66.73 & 67.00 & 0.45 & 0.018679 & 0.63 \\
\hline BookWorm & 40 & 38.57 & 39.00 & 1.50 & 33 & 40.50 & 40.50 & 2.06 & 0.000190 & $0.76 \boldsymbol{\Delta}$ \\
\hline Maniana & 40 & 49.73 & 49.50 & 1.76 & 31 & 50.33 & 50.00 & 1.56 & 0.059615 & $0.62 \Delta$ \\
\hline L9Droid & 40 & 55.40 & 55.00 & 1.73 & 31 & 56.40 & 56.50 & 1.69 & 0.010206 & $0.67 \boldsymbol{\Delta}$ \\
\hline Yaab & 40 & 27.83 & 28.00 & 0.38 & 38 & 28.03 & 28.00 & 0.32 & 0.017111 & $0.59 \boldsymbol{\Delta}$ \\
\hline Budget & 40 & 50.73 & 50.50 & 1.68 & 33 & 51.40 & 51.00 & 1.43 & 0.054662 & $0.62 \Delta$ \\
\hline Campyre & 40 & 13.90 & 14.00 & 0.92 & 31 & 15.00 & 15.00 & 0.59 & 0.000004 & $0.81 \boldsymbol{\Delta}$ \\
\hline URLazy & 40 & 32.00 & 32.00 & 0.00 & 32 & 32.00 & 32.00 & 0.00 & 1.000000 & $0.50 \triangleright$ \\
\hline ArXiv & 40 & 52.97 & 53.00 & 2.62 & 30 & 53.20 & 53.00 & 2.43 & 0.362501 & $0.53 \triangle$ \\
\hline Cetoolbox & 40 & 69.73 & 70.00 & 0.52 & 28 & 69.93 & 70.00 & 0.52 & 0.074087 & $0.59 \triangle$ \\
\hline CurrencyConverter & 40 & 62.00 & 62.00 & 2.18 & 30 & 62.87 & 63.00 & 1.57 & 0.079673 & $0.60 \Delta$ \\
\hline Charl & 40 & 73.60 & 74.00 & 0.50 & 26 & 73.57 & 74.00 & 0.50 & 0.400901 & $0.48 \nabla$ \\
\hline nverter & 40 & 39.10 & 39.00 & 1.69 & 31 & 39.53 & 40.00 & 1.31 & 51618 & $0.62 \Delta$ \\
\hline Anarx & 40 & 56.03 & 56.00 & 1.30 & 29 & 56.23 & 56.00 & 1.10 & 0.218234 & $0.56 \triangle$ \\
\hline Kindmind & 40 & 46.93 & 47.00 & 1.34 & 21 & 47.13 & 47.00 & 1.07 & 0.198322 & $0.56 \Delta$ \\
\hline Urforms & 40 & 63.13 & 63.00 & 1.80 & 32 & 63.77 & 63.00 & 1.83 & 0.100651 & $0.59 \triangle$ \\
\hline anager & 40 & 49.43 & 49.00 & 1.14 & 35 & 49.73 & 50.00 & 0.83 & 0.111142 & $0.59 \triangle$ \\
\hline Remembeer & 40 & 43.50 & 43.50 & 2.79 & 30 & 45.47 & 45.00 & 1.55 & 0.000381 & 0.75 А \\
\hline Pockettalk & 40 & 33.00 & 33.00 & 0.00 & 29 & 33.00 & 33.00 & 0.00 & 1.000000 & $0.50 \triangleright$ \\
\hline Rot13 & 40 & 71.00 & 71.00 & 0.00 & 31 & 71.00 & 71.00 & 0.00 & 1.000000 & $0.50 \triangleright$ \\
\hline IShows & 40 & 15.67 & 16.00 & 0.55 & 31 & 16.00 & 16.00 & 1.05 & 0.070921 & $0.59 \Delta$ \\
\hline Episc & 40 & 38.87 & 44.50 & 13.39 & 24 & 45.33 & 45.00 & 11.60 & 0.016614 & $0.66 \boldsymbol{\Delta}$ \\
\hline Angulo & 40 & 72.57 & 73.00 & 3.91 & 34 & 74.10 & 75.00 & 3.43 & 0.082998 & $0.60 \triangle$ \\
\hline Rightsalert & 40 & 78.33 & 78.00 & 0.48 & 35 & 78.50 & 78.50 & 0.51 & 0.098578 & $0.58 \triangle$ \\
\hline ApkTrack & 40 & 67.83 & 68.00 & 2.60 & 34 & 69.03 & 69.00 & 1.38 & 0.030447 & 0.64 А \\
\hline Diary & 40 & 75.43 & 75.00 & 0.77 & 32 & 75.33 & 75.00 & 0.61 & 0.322025 & $0.47 \nabla$ \\
\hline rtltcp & 40 & 36.63 & 37.00 & 2.20 & 28 & 36.97 & 37.00 & 1.96 & 0.341988 & $0.53 \triangle$ \\
\hline Fakedawn & 40 & 54.57 & 54.00 & 1.83 & 28 & 55.00 & 54.50 & 1.91 & 0.012839 & 0.65 스 \\
\hline Klaxon & 40 & 39.53 & 40.00 & 0.97 & 33 & 39.63 & 40.00 & 0.67 & 0.426840 & $0.51 \Delta$ \\
\hline
\end{tabular}

coverage by up to $7.14 \%$ compared to SAPIENZ ( $c f$. Campyre app). In absolute numbers, the increase of the median coverage is typically between 0.5 and 1.5 percentage points. Thus, the impact of the coverage improvement of SAPIENZ ${ }^{d i v}$ is rather slightly noticeable from a practical point of view. The remaining results for the 25/34 apps are inconclusive as they are not statistically significant. 
Answer to RQ2: Based on our evaluation of the coverage, we conclude that SAPIEnz ${ }^{\text {div }}$ significantly outperforms SAPIEnz on 9/34 apps, and SAPIENZ $^{\text {div }}$ was never significantly outperformed by SAPIENZ. From a practical perspective, SAPIENZ ${ }^{\text {div }}$ improves the median coverage by up to $7.14 \%$, in absolute numbers, however, by only up to 1.5 percentage points compared to SAPIENZ.

\subsubsection{Faults (RQ3)}

To compare the fault revelation capabilities of SAPIEnz ${ }^{d i v}$ and SAPIEnZ, we consider a crash of the app under test as a fault. Since the goal of testing is to find many unique crashes rather than to cause the same crash many times, the unique crashes are of particular importance when comparing the results. Thus, out of the total crashes we identify the unique crashes, that is, their stack traces are different from the stack traces of the other crashes of the same app caused by one approach. However, we exclude faults caused by Android (e.g., native crashes) and test harness (e.g., code instrumentation).

The corresponding results for the unique crashes revealed by SAPIENZ and SAPIENZ $^{\text {div }}$ for each of the 34 apps are shown in Table 8 that is structured similarly to Table 7. Again, we take the app-specific overhead of SAPIENz div into account ( $c f$. Section 5.3.1) and limit the number of generations for the search in SAPIENZ ${ }^{d i v}$ for each app. Overall, we obtained statistical significant results for $21 / 34$ apps. For 20 of these 21 apps, SAPIENZ ${ }^{\text {div }}$ significantly outperforms SAPIENZ with respect to the number of revealed unique crashes, in $10 / 20$ cases with a large effect size, in $6 / 20$ cases with a medium effect size, and in $4 / 20$ case with a small effect size. Concerning these results, specifically the median crashes, from a practical perspective, SAPIEnz ${ }^{\text {div }}$ was able to reveal up to 3 more unique crashes of an app than SAPIENZ ${ }^{\text {div }}$ ( $c f$. median crashes for the app ApkTrack). For the average crashes, SAPIENZ ${ }^{\text {div }}$ identified up to 3.74 more crashes of an app than SAPIENZ ( $c f$. average crashes for the app Campyre). On average across all apps, SAPIEnz ${ }^{\text {div }}$ identifies around one more unique crash of an app than SAPIENz. These results for 20/34 apps illustrate the practical impact SAPIENZ ${ }^{\text {div }}$ could have in revealing faults. In contrast, SAPIENZ significantly outperforms SAPIENZ ${ }^{\text {div }}$ for only one app (Pockettalk) with a very small effect size of 0.55 (or 0.45 from the perspective of SAPIEnZ $\left.{ }^{d i v}\right)$. For this app, none of the 30 SAPIENZ $^{\text {div }}$ runs revealed any crash, whereas 3/30 SAPIENZ runs revealed each a single crash. The results 
Table 8: Number of unique crashes revealed by SAPIEnZ after 40 generations (Gen.) and by SAPIENZ ${ }^{d i v}$ after fewer generations (Gen.) of search taking the app-specific overhead of SAPIENZ ${ }^{d i v}$ into account.

\begin{tabular}{|c|c|c|c|c|c|c|c|c|c|c|}
\hline Subject & \multicolumn{4}{|c|}{ SAPIENZ } & \multicolumn{4}{|c|}{ SAPIENZ ${ }^{d i v}$} & p-value & $\hat{A}_{12}$ \\
\hline BabyCare & 40 & 5.47 & 5.00 & 1.01 & 29 & 5.63 & 5.50 & 1.03 & 0.295757 & $0.54 \Delta$ \\
\hline Arity & 40 & 3.83 & 3.50 & 1.05 & 35 & 4.43 & 4.00 & 1.01 & 0.006799 & $0.68 \boldsymbol{\Lambda}$ \\
\hline JustSit & 40 & 3.73 & 3.00 & 1.23 & 35 & 3.67 & 3.00 & 0.92 & 0.429646 & $0.51 \triangle$ \\
\hline Hydrate & 40 & 0.63 & 0.00 & 0.89 & 33 & 0.80 & 1.00 & 0.85 & 0.180309 & $0.56 \Delta$ \\
\hline FillUp & 40 & 0.07 & 0.00 & 0.25 & 30 & 0.33 & 0.00 & 0.80 & 0.061647 & $0.57 \triangle$ \\
\hline Kanji & 40 & 3.47 & 3.00 & 1.04 & 31 & 3.73 & 4.00 & 0.87 & 0.154543 & $0.57 \triangle$ \\
\hline BookWorm & 40 & 2.17 & 2.00 & 1.12 & 33 & 2.47 & 2.00 & 1.41 & 0.254115 & $0.55 \triangle$ \\
\hline Maniana & 40 & 4.27 & 4.00 & 1.89 & 31 & 5.30 & 5.00 & 1.91 & 0.018722 & $0.65 \boldsymbol{\Lambda}$ \\
\hline L9Droid & 40 & 2.67 & 2.50 & 1.15 & 31 & 4.77 & 5.00 & 1.33 & 0.000000 & $0.88 \boldsymbol{\Lambda}$ \\
\hline Yaab & 40 & 1.57 & 1.00 & 0.68 & 38 & 1.87 & 1.50 & 1.17 & 0.237360 & $0.55 \Delta$ \\
\hline Budget & 40 & 6.23 & 6.00 & 0.97 & 33 & 7.20 & 7.00 & 0.92 & 0.000239 & $0.75 \boldsymbol{\Delta}$ \\
\hline Campyre & 40 & 2.73 & 3.00 & 0.74 & 31 & 6.47 & 4.00 & 6.76 & 0.000527 & 0.74 А \\
\hline URLazy & 40 & 0.07 & 0.00 & 0.25 & 32 & 0.03 & 0.00 & 0.18 & 0.285081 & $0.48 \nabla$ \\
\hline ArXiv & 40 & 2.60 & 3.00 & 0.89 & 30 & 4.90 & 4.00 & 2.17 & 0.000000 & 0.91 А \\
\hline Cetoolbox & 40 & 1.63 & 2.00 & 1.07 & 28 & 2.60 & 2.50 & 1.16 & 0.001144 & $0.72 \overline{\boldsymbol{\Lambda}}$ \\
\hline CurrencyConverter & 40 & 0.07 & 0.00 & 0.37 & 30 & 0.37 & 0.00 & 0.67 & 0.007549 & 0.61 А \\
\hline Charmap & 40 & 0.20 & 0.00 & 0.48 & 26 & 0.20 & 0.00 & 0.41 & 0.400048 & $0.51 \triangle$ \\
\hline onverter & 40 & 2.17 & 2.00 & 1.09 & 31 & 2.83 & 3.00 & 0.75 & 0.004628 & $0.68 \boldsymbol{\Delta}$ \\
\hline Anal & 40 & 1.60 & 1.00 & 1.30 & 29 & 3.57 & 3.00 & 2.13 & 0.000137 & $0.77 \overline{\boldsymbol{\Delta}}$ \\
\hline Kindmind & 40 & 7.00 & 7.00 & 2.12 & 21 & 8.37 & 8.00 & 2.11 & 0.000494 & $0.74 \boldsymbol{\Delta}$ \\
\hline Urforms & 40 & 5.53 & 6.00 & 1.01 & 32 & 7.00 & 7.00 & 1.93 & 0.000179 & $0.76 \boldsymbol{\Delta}$ \\
\hline Homen & 40 & 11.13 & 11.00 & 1.20 & 35 & 11.60 & 11.50 & 1.16 & 0.111629 & $0.59 \triangle$ \\
\hline Remembeer & 40 & 1.67 & 1.50 & 0.76 & 30 & 2.10 & 2.00 & 0.92 & 0.032226 & 0.63 А \\
\hline Pockettalk & 40 & 0.10 & 0.00 & 0.31 & 29 & 0.00 & 0.00 & 0.00 & 0.040702 & $0.45 \boldsymbol{\nabla}$ \\
\hline Rot13 & 40 & 0.07 & 0.00 & 0.37 & 31 & 0.20 & 0.00 & 0.41 & 0.028777 & $0.58 \boldsymbol{\Delta}$ \\
\hline DroidShows & 40 & 0.30 & 0.00 & 0.47 & 31 & 1.97 & 1.00 & 2.75 & 0.000424 & 0.73 А \\
\hline Episo & 40 & 0.13 & 0.00 & 0.43 & 24 & 0.50 & 0.00 & 0.90 & 0.015 & $0.62 \boldsymbol{\Lambda}$ \\
\hline & 40 & 0.97 & 1.00 & 0.76 & 34 & 1.53 & 1.00 & 1.07 & 0.016776 & 0.65 А \\
\hline Rightsalert & 40 & 13.90 & 14.00 & 2.04 & 35 & 15.17 & 15.00 & 1.82 & 0.003657 & $0.70 \boldsymbol{\Delta}$ \\
\hline ApkTrack & 40 & 20.00 & 20.00 & 3.52 & 34 & 23.00 & 23.00 & 3.22 & 0.000317 & $0.76 \boldsymbol{\Lambda}$ \\
\hline & 40 & 3.10 & 3.00 & 0.31 & 32 & 3.27 & 3.00 & 0.52 & 0.081530 & $0.57 \triangle$ \\
\hline & 40 & 1.63 & 1.00 & 0.81 & 28 & 1.87 & 2.00 & 0.68 & 0.077531 & $0.60 \triangle$ \\
\hline Fakedawn & 40 & 0.17 & 0.00 & 0.46 & 28 & 0.27 & 0.00 & 0.52 & 0.172190 & $0.55 \triangle$ \\
\hline Klaxon & 40 & 0.83 & 1.00 & 0.79 & 33 & 1.43 & 1.50 & 0.90 & 0.004092 & 0.69 А \\
\hline
\end{tabular}

for the remaining 13/34 apps are not statistically significant and therefore inconclusive.

Finally, concerning the total number of apps and the whole experiment (particularly all repetitions of runs), SAPIENZ was able to reveal faults in 34/34 apps, whereas SAPIEnZ ${ }^{\text {div }}$ was able to reveal faults in 33/34 apps. 
Answer to RQ3: Based on our evaluation of the crashes, we conclude that SAPIENZ ${ }^{\text {div }}$ significantly outperforms SAPIENZ on 20/34 apps, and SAPIENZ significantly outperforms SAPIENZ ${ }^{\text {div }}$ on $1 / 34$ apps. From a practical perspective, SAPIENZ ${ }^{\text {div }}$ was able to reveal up to 3 and 3.74 more unique crashes of an app than SAPIENZ considering the median and average crashes, respectively.

\subsubsection{Length of Fault-Revealing Test Sequences (RQ4)}

To analyze the length of test sequences (cases), we consider only those test sequences that reveal faults similarly to [1]. Moreover, to measure the length of such sequences within one run of an approach on an app, we consider the minimal length, that is, the length of the shortest of all sequences produced in this run causing the same unique crash. If more than one unique crash is found within one run, we take the average length of the corresponding minimal test sequences.

The corresponding results showing the average length of the minimal fault-revealing test sequences produced by SAPIENZ and SAPIENZ ${ }^{d i v}$ are presented in Table 9. As before, we take the app-specific overhead of SAPIENZ ${ }^{d i v}$ into account ( $c f$. Section 5.3.1) and limit the number of generations for the search in SAPIENZ ${ }^{d i v}$ for each app. If no fault has been identified for an app by an approach in any of the 30 runs, there is no fault-revealing test sequence as denoted by '-' for the mean, median, SD, p-value, and $\hat{A}_{12}$ columns. This is only the case for SAPIENZ ${ }^{d i v}$ and Pockettalk. Moreover, if exactly one out of the 30 runs of an approach on an app has revealed faults, there is no standard deviation across the runs, which is denoted by '-' in the SD column while there exists mean and median values.

Concerning the results of Table 9, we obtained statistical significant results for 16/34 apps. For all of these 16 apps, SAPIENZ significantly outperformed SAPIENZ ${ }^{d i v}$ by producing shorter fault-revealing test sequences, in $11 / 16$ cases with a large effect size, in 2/16 with a medium effect size, and in $3 / 16$ cases with a small effect size. Considering these significant results, the median length of fault-revealing test sequences produced by SAPIENZ ${ }^{d i v}$ is at least $10.75 \%$ (cf. Homemanager app) and up to $118.10 \%$ ( $c f$. BookWorm app) longer than the median length of corresponding sequences produced by SAPIENZ. In absolute numbers, this translates to an increase of the median length of fault-revealing test sequences by 18.5 events for the Homemanager 
Table 9: Average length of the minimal fault-revealing test sequences produced by SAPIENZ after 40 generations (Gen.) and by SAPIENZ ${ }^{d i v}$ after fewer generations (Gen.) of search taking the app-specific overhead of SAPIENZ ${ }^{\text {div }}$ into account.

\begin{tabular}{|c|c|c|c|c|c|c|c|c|c|c|}
\hline Subject & \multicolumn{4}{|c|}{ SAPIENZ } & \multicolumn{4}{|c|}{ SAPIENZ ${ }^{d i v}$} & p-value & $\hat{A}_{12}$ \\
\hline BabyCare & 40 & 71.53 & 61.00 & 36.78 & 29 & 112.50 & 119.00 & 50.88 & 0.001024 & $0.27 \mathbf{\nabla}$ \\
\hline Arity & 40 & 100.87 & 100.50 & 41.51 & 35 & 154.90 & 162.00 & 46.63 & 0.000027 & $0.20 \mathbf{\nabla}$ \\
\hline JustSit & 40 & 216.13 & 213.00 & 61.09 & 35 & 209.83 & 211.00 & 69.31 & 0.432492 & $0.51 \Delta$ \\
\hline Hydrate & 40 & 296.69 & 301.00 & 146.72 & 33 & 321.75 & 334.00 & 105.42 & 0.421762 & $0.48 \nabla$ \\
\hline FillUp & 40 & 338.50 & 338.50 & 78.49 & 30 & 325.00 & 365.00 & 147.19 & 0.433816 & $0.50 \triangleright$ \\
\hline Kanji & 40 & 74.33 & 80.50 & 50.89 & 31 & 136.17 & 135.50 & 51.86 & 0.000027 & $0.20 \mathbf{\nabla}$ \\
\hline BookWorm & 40 & 101.57 & 58.00 & 88.40 & 33 & 141.67 & 126.50 & 103.73 & 0.040507 & $0.37 \mathbf{\nabla}$ \\
\hline Maniana & 40 & 306.30 & 309.00 & 59.89 & 31 & 354.67 & 355.00 & 53.94 & 0.001516 & 0.28 \\
\hline L9Droid & 40 & 282.62 & 278.00 & 99.52 & 31 & 312.63 & 317.00 & 64.93 & 0.092302 & $0.40 \nabla$ \\
\hline Yaab & 40 & 135.73 & 119.50 & 63.63 & 38 & 151.00 & 116.00 & 88.03 & 0.412239 & $0.48 \nabla$ \\
\hline Budget & 40 & 243.07 & 241.00 & 44.56 & 33 & 240.57 & 237.00 & 49.69 & 0.336705 & $0.53 \triangle$ \\
\hline Campyre & 40 & 108.43 & 91.00 & 57.23 & 31 & 161.73 & 169.00 & 84.70 & 0.007978 & $0.32 \nabla$ \\
\hline URLazy & 40 & 254.00 & 254.00 & 315.37 & 32 & 484.00 & 484.00 & & 0.270146 & $0.00 \nabla$ \\
\hline ArXiv & 40 & 178.63 & 172.00 & 87.69 & 30 & 234.83 & 214.50 & 65.53 & 0.002062 & $0.28 \boldsymbol{\nabla}$ \\
\hline Cetoolbox & 40 & 171.32 & 161.00 & 79.64 & 28 & 273.62 & 268.00 & 84.40 & 0.000051 & $0.19 \boldsymbol{\nabla}$ \\
\hline CurrencyConverter & 40 & 271.00 & 271.00 & & 30 & 411.38 & 425.50 & 62.10 & 0.087622 & $0.00 \nabla$ \\
\hline Charmap & 40 & 408.80 & 434.00 & 100.18 & 26 & 400.00 & 460.50 & 153.70 & 0.463632 & $0.50 \triangleright$ \\
\hline Nanc & 40 & 121.81 & 124.00 & 75.55 & 31 & 210.33 & 195.00 & 84.96 & 0122 & $0.22 \boldsymbol{\nabla}$ \\
\hline Anar & 40 & 277.30 & 272.00 & 97.36 & 29 & 292.64 & 296.00 & 94.25 & 0.345483 & $0.47 \nabla$ \\
\hline Kindmind & 40 & 150.87 & 154.00 & 44.46 & 21 & 213.53 & 214.50 & 42.70 & 0.000002 & $0.15 \mathbf{v}$ \\
\hline Urforms & 40 & 219.50 & 224.00 & 54.10 & 32 & 243.17 & 232.00 & 51.81 & 0.077853 & $0.39 \nabla$ \\
\hline Hom & 40 & 176.10 & 172.00 & 37.21 & 35 & 194.17 & 190.50 & 42.03 & 2476 & $0.37 \mathbf{v}$ \\
\hline Remembeer & 40 & 122.60 & 102.00 & 65.16 & 30 & 173.17 & 205.00 & 99.95 & 0.031761 & $0.36 \boldsymbol{\nabla}$ \\
\hline Pockettalk & 40 & 85.67 & 57.00 & 61.26 & 29 & & - & - & - & - \\
\hline Rot13 & 40 & 73.00 & 73.00 & & 31 & 288.50 & 289.00 & 175.85 & 0.105650 & $0.00 \nabla$ \\
\hline DroidShows & 40 & 160.56 & 147.00 & 98.98 & 31 & 268.15 & 251.00 & 126.50 & 0.012572 & $0.23 \boldsymbol{\nabla}$ \\
\hline Episodes & 40 & 381.00 & 414.00 & 91.10 & 24 & 356.40 & 430.50 & 157.00 & 0.432795 & $0.45 \nabla$ \\
\hline & 40 & 335.14 & 353.00 & 84.12 & 34 & 311.81 & 318.00 & 94.29 & 0.218887 & $0.57 \Delta$ \\
\hline Rightsalert & 40 & 203.10 & 210.00 & 33.79 & 35 & 232.30 & 237.50 & 37.21 & 0.000450 & $0.25 \mathbf{\nabla}$ \\
\hline ApkTrack & 40 & 199.90 & 200.00 & 33.90 & 34 & 246.73 & 251.50 & 25.86 & 0.000000 & 0.13 \\
\hline Diary & 40 & 109.37 & 109.50 & 34.68 & 32 & 139.10 & 125.50 & 43.29 & 0.004628 & $0.30 \boldsymbol{\nabla}$ \\
\hline & 40 & 114.97 & 68.00 & 88.01 & 28 & 153.70 & 163.50 & 98.59 & 0.100453 & $0.40 \nabla$ \\
\hline Fakedawn & 40 & 219.75 & 170.50 & 192.32 & 28 & 273.29 & 302.00 & 162.47 & 0.318301 & $0.39 \nabla$ \\
\hline Klaxon & 40 & 315.79 & 338.00 & 122.95 & 33 & 354.52 & 360.00 & 84.12 & 0.187113 & $0.42 \nabla$ \\
\hline
\end{tabular}

app (median length increased from 172.0 to 190.5 events) and 68.5 events for the BookWorm app (median length increased from 58.0 to 126.5 events). In contrast, SAPIENZ ${ }^{\text {div }}$ was not able to significantly outperform SAPIENZ on any app for the length of fault-revealing test sequences. The results for the remaining 18/34 apps are not statistically significant and therefore inconclusive. 
Answer to RQ4: Based on our evaluation of the length of minimal fault-revealing test sequences, we conclude that SAPIENZ significantly outperforms SAPIENZ $^{\text {div }}$ on 16/34 apps by producing shorter test sequences, whereas SAPIENZ ${ }^{\text {div }}$ was not able to significantly outperform SAPIENZ on any app. From a practical perspective, the fault-revealing sequences of SAPIENZ ${ }^{\text {div }}$ are at least $10.75 \%$ and up to $118.10 \%$ longer than the corresponding sequences of SAPIENZ for an app.

\subsection{Discussion}

The diversity-promoting mechanisms of SAPIENZ ${ }^{d i v}$ add a significant runtime overhead to SAPIENZ ranging from $5.47 \%$ to $46.57 \%$ for individual apps when searching over 40 generations. The worse execution time of SAPIENZ ${ }^{d i v}$ is caused by two factors. On the one hand, the diversity-promoting mechanisms of SAPIENZ ${ }^{d i v}$ result in a runtime overhead, for instance, due to the more costly creation of the initial population when twice the number of individuals are generated and evaluated, and to the computation of the diversity of the population in each generation. On the other hand, SAPIEnZ ${ }^{d i v}$ produces diverse test sequences that are inherently longer than the test sequences produced by SAPIENZ. Thus, executing longer sequences on the app under test during fitness evaluation takes more time than for shorter test sequences.

Given this runtime overhead of SAPIENZ ${ }^{d i v}$, to fairly compare SAPIENZ ${ }^{\text {div }}$ and SAPIENZ, the search of SAPIENZ ${ }^{d i v}$ has to be done with a smaller number of generations than the search of SAPIENZ. Thus, in the context of comparing the results of our empirical study, we reduced the number of generations used by the search of SAPIENZ ${ }^{d i v}$ according to the app-specific overhead, whereas the search of SAPIENZ can use all of the 40 generations. This adjustment gives both approaches the same time budget and therefore allows us to compare fairly the achieved coverage, revealed faults, and length of minimal fault-revealing test sequences of both approaches.

SAPIENZ $^{\text {div }}$ significantly outperforms SAPIENZ on 9/34 apps for coverage, on 20/34 apps for faults, and on 0/34 apps for length. In contrast, SAPIENZ significantly outperforms SAPIENZ ${ }^{d i v}$ on $0 / 34$ apps for coverage, on $1 / 34$ apps for faults, and on 16/34 apps for length. The remaining results are not statistically significant and therefore inconclusive.

Thus, SAPIENZ ${ }^{d i v}$ achieves better or at least similar test results for coverage and faults than SAPIENZ, so that preferring SAPIENZ ${ }^{d i v}$ over SAPIENZ for 
app testing will likely not result in disadvantages concerning fault revelation and coverage. Consequently, promoting diversity can be beneficial for generating test suites for apps. In practical terms, SAPIENZ ${ }^{\text {div }}$ revealed up to 3 and 3.74 more unique crashes of an app than SAPIENZ considering the median and average crashes, respectively. On average across all apps, SAPIENZ ${ }^{\text {div }}$ identified around one more unique crash of an app than SAPIENz. This illustrates the impact on fault revelation that SAPIENZ ${ }^{\text {div }}$ could have in practice. In contrast, the practical impact of the improved coverage achieved by SAPIENZ $^{\text {div }}$ is less noticeable. SAPIEnz ${ }^{\text {div }}$ was able to increase the median coverage for an individual app by up to $7.14 \%$ compared to SAPIENZ, in absolute numbers, however, by only up to 1.5 percentage points.

However, SAPIEnZ ${ }^{\text {div }}$ performs worse than SAPIENZ in producing short fault-revealing test sequences for 16/34 apps. For the remaining 18/34 apps, we did not observe a statistically significant difference between lengths of such sequences produced by SAPIEnz ${ }^{d i v}$ and SAPIENZ. Thus, promoting diversity in SAPIENZ ${ }^{d i v}$ tends to result in test sequences of similar or greater length. A corresponding observation has been made for diversity in unit testing resulting in longer test cases [37]. We think that the reason for this observation is that the search can easily achieve a higher diversity between test sequences by exploring longer sequences. That is, the space of possible test sequences grows with the sequence length, and likewise the probability of generating distant sequences grows with the increase of the sequence length. Considering the 16/34 apps, for which we obtained statistically significant results for length, the median length of fault-revealing test sequences produced by SAPIENZ ${ }^{d i v}$ for an app is longer than the median length of corresponding sequences produced by SAPIENZ, by at least $10.75 \%$ and up to $118.10 \%$. Thus, the growth of the sequence length in SAPIENZ ${ }^{\text {div }}$ could be acceptable but it may also result in sequences being more than double the length of the sequences produced by SAPIENZ.

Consequently, when selecting SAPIEnz ${ }^{\text {div }}$ over SAPIEnz, a trade-off has to be made that favors potentially improved fault revelation and coverage results while accepting potentially longer test sequences that developers have to understand to debug the app and fix the fault in the app. From a practical point of view, the improved fault revelation capabilities of SAPIENZ ${ }^{\text {div }}$ are most promising since SAPIENZ ${ }^{\text {div }}$ was able to find more unique crashes for most of the apps than SAPIENZ (up to 3 more crashes of an app considering the median crashes). In contrast, the improved coverage achieved by SAPIENZ ${ }^{\text {div }}$ seems to be less relevant in practice as the improvement is 
rather marginal in absolute numbers (up to 1.5 percentage points for the median coverage of an app). Thus, to adopt SAPIENZ ${ }^{\text {div }}$ in practice, a tradeoff needs to be primarily made between the improved fault revelation and the additional costs for developers to understand and debug longer test sequences. Such a trade-off might particularly favor SAPIENZ ${ }^{\text {div }}$ over SAPIENZ in cases where the increase of the sequence length is likely to be acceptable, for instance, by being only around $11 \%$.

\section{Threats to Validity}

Internal validity. A threat to the internal validity is a bias in the selection of the five apps from $[1,16]$ for the fitness landscape analysis, and of the nine apps from Mao et al. [1], 21 apps from $\mathrm{Su}$ et al. [32], and four further apps we choose randomly from F-Droid for the evaluation of SAPIENz ${ }^{\text {div }}$. We mitigated this threat by using apps from different sources: three different research papers, while Choudhary et al. [16] collected their apps from four different papers, and a random sampling from F-Droid.

To reduce the threat of overfitting to the given apps, we use the default configuration of SAPIENZ and SAPIENZ ${ }^{\text {div }}$ without any parameter tuning. Moreover, we conservatively set the parameters that control the diversitypromoting mechanisms in SAPIENZ ${ }^{\text {div }}$, again without any parameter tuning (cf. Section 5.2). However, a less conservative parameter setting enforcing diversity earlier and stronger might lead to other results.

The correctness of the diversity-promoting mechanisms is a threat that we addressed by computing the fitness landscape analysis metrics with SAPIENZ ${ }^{\text {div }}$ to confirm the improved diversity. Finally, the choice of distance metric for test suites is a threat that we addressed by following recommendations from fitness landscape analysis research ( $c f$. Sections 2.2 and 3.1). Still, the results of the fitness landscape analysis and evaluation of SAPIEnz ${ }^{d i v}$ might be different when using another metric (e.g., edit distance).

External validity. As we used five different Android apps for analyzing the fitness landscape of SAPIENZ and 34 different apps for evaluating SAPIENZ ${ }^{\text {div }}$ out of over 2,500 apps on F-Droid and millions on Google Play, this small sample size is a potential threat to the generalizability of our findings. To strengthen external validity, a larger number of experiments could be applied to more apps, for instance, to the 68 apps of the F-Droid benchmark by Choudhary et al. [16], which, however, would imply large evaluation costs in terms of computation time. 
Construct validity. A threat to construct validity are the measures that we use to compare SAPIENZ and SAPIEnz ${ }^{\text {div }}$. Since the goal of testing is to find faults, we do not measure faults directly but crashes of the app caused by uncaught exceptions. We believe that this approach is justified as we consider system-level end-to-end testing, that is, tests mimic end user behavior and there should not be any uncaught exception in an app from the end user perspective. As further measures, we consider coverage and the length of test sequences. In this context, we do not know how coverage and fault revelation are correlated, however, coverage as a secondary criterion for evaluation testing approaches is an accepted measure [38]. Moreover, we do not know which lengths of test sequences are acceptable for developers who use such sequences to debug an app. Thus, the length of a test sequence might not be meaningful when using it as the only measure to evaluate a testing approach. Consequently, we use the triple of crashes, coverage, and sequence length as measures, which are also the objectives of the fitness function of SAPIENZ/SAPIENZ ${ }^{d i v}$ and thus, has been used to evaluate SAPIENZ [1].

Statistical and conclusion validity. A threat to statistical and conclusion validity is our choice of statistical test (Mann-Whitney U [33]) and the effect size measure (Vargha-Delaney [36]). Having data for two levels (SAPIENZ and SAPIEnZ ${ }^{\text {div }}$ ) and independent sample sets (based on 30 runs of an approach on an app), we compare both approaches for each individual app. We further cannot make any assumption about the distribution of the results. All these aspects justify our choice of the Mann-Whitney U test. Moreover, we use the Vargha-Delaney effect size measure $\hat{A}_{12}$ to compare SAPIENZ ${ }^{\text {div }}$ and SAPIENZ as recommended by Arcuri and Briand [34]. This measure is a probability estimate denoting "how likely is it that an investigated technique $X\left[\right.$ e.g., SApIEnZ $\left.{ }^{d i v}\right]$ is better than technique $Y$ [e.g., SAPIEnZ]" [35, p. 261]. A threat to conclusion validity are the probability thresholds for the effect size to distinguish large, medium, and small improvements of one technique over the other. We use the thresholds proposed by Vargha and Delaney [36] and used by Mao et al. [1] to evaluate SAPIENZ.

\section{Related Work}

Related work can be identified in three main areas: approaches on test case generation for apps, approaches on diversity in search-based software testing, and fitness landscape analysis for software engineering problems. 
Test case generation for mobile applications. Strategies for generating test cases can be grouped into approaches using random, model- and searchbased, or systematic exploration strategies for the generation.

Random strategies such as Monkey [30] implement UI-guided test input generators where events on the GUI are selected randomly. Dynodroid [31] extends the random selection using weights and frequencies of events. Modeland search-based strategies such as COBWEB [39], SwiftHand [40], EHBDroid [41], PUMA [42], EvoDroid [43], DroidBot [44], MobiGUITAR [45], juGULAR [46], ABE [47], Humanoid [48], SAPIEnz [1], Stoat [32], and MATE [9] apply model-based testing with dedicated search strategies to mobile applications. The type of model and search strategy is different in each approach and can range from simple to sophisticated solutions.

For instance, COBWEB [39] extracts a graph-based energy model which is then used in combination with a genetic algorithm to construct test cases to reveal so called energy bugs. Similar evolutionary exploration strategies are used by EvoDroid [43] and Sapienz [1]. SwiftHand [40] on the other hand uses approximate learning to learn the app's GUI model during testing. The learned GUI model enables generation of inputs that visit unexplored states of the app. Alternatively, juGULAR [46] uses machine learning as an automatic GUI model exploration engine. Thus, systematic exploration strategies apply full-scale symbolic execution [49] to evolutionary algorithms. All of these approaches do not explicitly manage diversity, except of Stoat [32] that encodes the diversity of sequences into the objective function.

Diversity in search-based software testing. The diversity of solutions has been researched in search-based software testing, mostly in the area of test case selection and generation. For test case selection, Panichella et al. [14] showed that multi-objective genetic algorithms (GAs) can be significantly improved by diversifying the solutions in the search process. They propose several diversity-preserving techniques for the main loop of NSGA-II. The resulting algorithm is called DIV-GA (DIVersity based GA). The conducted study showed that DIV-GA outperforms both NSGA-II and vNSGA-II [50] that are considered state of the art for multi-objective optimization.

For test case generation, there are approaches that consider feature diversity $[51,52,37]$ in their search procedures. The closest to our approach is the work of Biagiola et al. [51] that applies diversity-based test case generation to web applications. The results show that diversity-enabled search-based testing approaches achieve a higher state coverage, code coverage, and fault 
detection rate than random test case generation approaches. The work of Feldt and Poulding [52] experiments with ten different search strategies that are build on top of GÖDELTEST [53] which is a search-based data generation framework. The results show that spread-aware random sampling such as latin hypercube sampling is very effective in creating diverse test cases. Additionally, the study of Albunian [37] matches our observation that promoting diversity during the test case generation process will increase the length of tests without improving considerably the coverage.

Current approaches for test case selection and generation witness that diversity promotion is crucial although its realization "requires some care" $[17$, p. 782]. Finally, the suitability of metrics for diversity of a test suite [54] and the visualization of test diversity [55] are still open research topics.

Fitness landscape analysis for software engineering problems. Several approaches have analyzed fitness landscapes of various search-based software engineering problems. For search-based testing, for instance, Waeselynck et al. [56] investigate the ruggedness (local structure) and size of the search space to configure a simulated annealing algorithm for test generation. The size of the search space is quantified by a diameter metric, whereas we use several diameter metrics to measure the distance between individuals of a population. Lefticaru and Ipate [57] investigate the local structure and size of the search space similarly to Waeselynck et al. [56] and extend their analysis with a problem hardness measure (fitness distance correlation) that, however, requires knowledge about the global optimum, which is, for instance, not known for the problem of testing apps. Based on their analysis, various fitness functions are evaluated for specification-based testing.

More recently, Aleti et al. [58] have analyzed the evolvability and local structure of the landscape for the problem of generating whole test suites with EvoSuite. The fitness landscape analysis used three metrics, namely population information content, negative slope coefficient, and change rate, to mainly assess solvability. Based on their analysis, they conclude that "the search space is rather poor in gradients to local optima" [58, p.619] and it is either unimodal or has few modes with many plateaus. In the same direction, Albunian et al. [59] further analyze the local structure in terms of ruggedness and neutrality of the landscape for generating tests with the MOSA (Many-Objective Sorting Algorithm) version of EvoSuite. Similarly to Aleti et al. [58], their analysis showed that the landscape is dominated by plateaus. Our two metrics for evolvability ppos and $h v$ confirm this obser- 
vation. Additionally, our fitness landscape analysis complements the studies of Aleti et al. [58] and Albunian et al. [59] with a detailed analysis of the diversity of the population and the diversity of the pareto-optimal solutions, which shows a drastically decreasing diversity over time.

Besides search-based testing, a fitness landscape analysis has been conducted for other search-based software engineering problems. For instance, Aleti and Moser [60] tackle the problem of optimizing software architectures while focusing on an analysis of the local structure (ruggedness). Another line of research analyzes the fitness landscape to identify whether the landscape is elementary or not [61], or to construct elementary landscapes [62]. Such landscapes are a special class of fitness landscapes that could inform the development of suitable heuristics, for instance, for the next release problem [61] or test suite minimization [62].

In contrast to our work, none of these approaches address the problem of generating test suites for mobile apps or focus on the global topology, that is, how solutions and fitnesses are spread in the search space in terms of diversity of dominated and non-dominated solutions being evolved. This makes our previous work [7] extended in this article novel.

\section{Conclusions and Future Work}

In this article, we reported on our descriptive study analyzing the fitness landscape of SAPIENZ indicating a lack of diversity of test suites being evolved during the search. Therefore, we proposed SAPIEnz ${ }^{\text {div }}$ that integrates four mechanisms to promote diversity. The evaluation shows that SAPIENZ ${ }^{d i v}$ achieves better or at least similar test results in terms of achieved coverage and revealed faults than SAPIENZ, but it generates fault-revealing test sequences of similar or greater length than SAPIEnz. Thus, preferring SAPIENZ $^{\text {div }}$ over SAPIENZ for app testing could be advantageous concerning fault revelation and coverage, and disadvantageous concerning the length of test sequences that developers have to understand to debug the app and fix the fault in the app. Thus, the understanding of the search problem obtained by the fitness landscape analysis helped us to find a more suitable configuration of SAPIENZ without trial-and-error experiments. Particularly, we think that the costs of performing the fitness landscape analysis with five apps and

five repetitions would be lower than performing trial-and-error experiments with different SAPIENZ configurations to gain the same insights. 
This illustrates the general benefits a fitness landscape analysis could have for search-based software engineering. By investigating the fitness landscape, characteristics and particularly difficulties of a search problem (e.g., a decreasing diversity of solutions being evolved [7], or many plateaus in the landscape $[58,59]$ ) could be identified. Such analytical results enable a better understanding of the search problem and search algorithm, whose behavior is otherwise a black box [11]. Moreover, the improved understanding could be leveraged to develop more suitable algorithms (e.g., in terms of operators or parameter configurations) for the given problem, especially algorithms that can cope with the identified difficulties. Without a fitness landscape analysis, improved algorithms are typically identified by empirically trying out variants of algorithms, which is a trial-and-error and costly process. With the gained understanding from a fitness landscape analysis, such a process should be avoided and the development of algorithms should be systemized.

Practitioners such as software testers would benefit from a fitness landscape analysis as it informs them on how to improve or at least appropriately configure search-based testing tools (e.g., considering the many configuration parameters of state-of-the-art heuristics) for their problem at hand. However, in practice and research the challenge arises of how to conduct a fitness landscape analysis because many aspects aspects of fitness landscape could be analyzed (e.g., the global topology, local structure, and evolvability) with many metrics $[4,5]$. This calls for developing guidelines for software engineering researchers and practitioners of how to conduct fitness landscape analyses and for integrating corresponding mechanisms into search-based tools.

Similar to the effectiveness mapping of automated test suite generation techniques by Oliveira et al. [63], we plan to analyze the apps on which SAPIENZ and SAPIENZ ${ }^{\text {div }}$ perform differently to identify and map app characteristics for which a high diversity of test suites is beneficial for a search. Such characteristics could be the criteria to select SAPIENZ ${ }^{\text {div }}$ - when diversity is a concern - at the costs of longer execution times, otherwise SAPIENZ as the more time efficient approach would be chosen. Finally, we plan to investigate other parameter settings controlling the novel SAPIENZ ${ }^{\text {div }}$ features to enforce diversity earlier and stronger.

Acknowledgments This work has been developed in the FLASH project (GR 3634/6-1) funded by the German Science Foundation (DFG) and has been partially supported by the 2018 Facebook Testing and Verification research award. We thank the Hasso Plattner Institute for providing us access to the HPI Future SOC computing infrastructure to conduct the experiments. 


\section{References}

[1] K. Mao, M. Harman, Y. Jia, Sapienz: Multi-objective automated testing for android applications, in: Proc. of the 25th International Symposium on Software Testing and Analysis, ISSTA, ACM, 2016, pp. 94-105.

[2] G. Fraser, A. Arcuri, Whole test suite generation, IEEE Transactions on Software Engineering 39 (2013) 276-291.

[3] A. Arcuri, G. Fraser, Parameter tuning or default values? an empirical investigation in search-based software engineering, Empirical Software Engineering 18 (2013) 594-623.

[4] K. M. Malan, A. P. Engelbrecht, A survey of techniques for characterising fitness landscapes and some possible ways forward, Information Sciences 241 (2013) 148-163.

[5] E. Pitzer, M. Affenzeller, A comprehensive survey on fitness landscape analysis, in: Recent Advances in Intelligent Engineering Systems, Springer, 2012, pp. 161-191.

[6] I. Moser, M. Gheorghita, A. Aleti, Identifying features of fitness landscapes and relating them to problem difficulty, Evolutionary Computation 25 (2017) 407-437.

[7] T. Vogel, C. Tran, L. Grunske, Does diversity improve the test suite generation for mobile applications?, in: 11th Intl. Symposium on SearchBased Software Engineering, SSBSE, Springer, 2019, pp. 58-74.

[8] K. Deb, A. Pratap, S. Agarwal, T. Meyarivan, A fast and elitist multiobjective genetic algorithm: NSGA-II, Transactions on Evolutionary Computation 6 (2002) 182-197.

[9] L. Sell, M. Auer, C. Frädrich, M. Gruber, P. Werli, G. Fraser, An empirical evaluation of search algorithms for app testing, in: Proc. of the IFIP International Conference on Testing Software and Systems, Springer, 2019, pp. 123-139.

[10] M. Črepinšek, S.-H. Liu, M. Mernik, Exploration and exploitation in evolutionary algorithms: A survey, ACM Comput. Surv. 45 (2013) 35:135:33.

[11] J. C. Culberson, On the futility of blind search: An algorithmic view of "no free lunch", Evol. Comput. 6 (1998) 109-127.

[12] P. F. Stadler, Fitness landscapes, in: Biological Evolution and Statistical Physics, Springer, 2002, pp. 183-204. 
[13] T. Jones, Evolutionary Algorithms, Fitness Landscapes and Search, Ph.D. thesis, The University of New Mexico, Albuquerque, NM, 1995.

[14] A. Panichella, R. Oliveto, M. D. Penta, A. D. Lucia, Improving multiobjective test case selection by injecting diversity in genetic algorithms, IEEE Trans. Software Eng. 41 (2015) 358-383.

[15] T. Smith, P. Husbands, P. J. Layzell, M. O'Shea, Fitness landscapes and evolvability, Evolutionary Computation 10 (2002) 1-34.

[16] S. R. Choudhary, A. Gorla, A. Orso, Automated test input generation for android: Are we there yet?, in: Proc. of the 30th Int. Conference on Automated Software Engineering (ASE), IEEE, 2015, pp. 429-440.

[17] R. C. Purshouse, P. J. Fleming, On the evolutionary optimization of many conflicting objectives, IEEE Transactions on Evolut. Comp. 11 (2007) 770-784.

[18] M. Li, X. Yao, Quality evaluation of solution sets in multiobjective optimisation: A survey, ACM Comput. Surv. 52 (2019) 26:1-26:38.

[19] S. Wang, S. Ali, T. Yue, Y. Li, M. Liaaen, A practical guide to select quality indicators for assessing pareto-based search algorithms in searchbased software engineering, in: Proceedings of the 38th International Conference on Software Engineering, ICSE, ACM, 2016, pp. 631-642.

[20] V. Bachelet, Métaheuristiques Parallèles Hybrides: Application au Problème D'affectation Quadratique, Ph.D. thesis, Université Lille-I, 1999.

[21] O. Olorunda, A. P. Engelbrecht, Measuring exploration/exploitation in particle swarms using swarm diversity, in: Proc. of the Congress on Evolutionary Computation (IEEE World Congress on Computational Intelligence), IEEE, 2008, pp. 1128-1134.

[22] H. Isermann, The enumeration of the set of all efficient solutions for a linear multiple objective program, Operational Research Quarterly 28 (1977) 711-725.

[23] L. Paquete, T. Stützle, Clusters of non-dominated solutions in multiobjective combinatorial optimization: An experimental analysis, in: Multiobjective Programming and Goal Programming, Springer, 2009, pp. 69-77.

[24] A. Liefooghe, S. Verel, H. Aguirre, K. Tanaka, What makes an instance difficult for black-box 0-1 evolutionary multiobjective optimizers?, in: 
Artificial Evolution: 11th Int. Conference, Evolution Artificielle, EA 2013, Springer, 2014, pp. 3-15.

[25] J. Morrison, F. Oppacher, Maintaining genetic diversity in genetic algorithms through co-evolution, in: Advances in Artificial Intelligence: 12th Biennial Conference of the Canadian Society for Computational Studies of Intelligence, AI'98, Springer, 1998, pp. 128-138.

[26] O. M. Shir, M. Preuss, B. Naujoks, M. Emmerich, Enhancing decision space diversity in evolutionary multiobjective algorithms, in: Evolutionary Multi-Criterion Optimization, Springer, 2009, pp. 95-109.

[27] H. Maaranen, K. Miettinen, A. Penttinen, On initial populations of a genetic algorithm for continuous optimization problems, Journal of Global Optimization 37 (2006) 405-436.

[28] S. Ronald, Duplicate genotypes in a genetic algorithm, in: Proc. of the International Conference on Evolutionary Computation. IEEE World Congress on Computational Intelligence, IEEE, 1998, pp. 793-798.

[29] S. Y. Yuen, C. K. Chow, A genetic algorithm that adaptively mutates and never revisits, IEEE Transactions on Evolutionary Computation 13 (2009) 454-472.

[30] Android, Ui/application exerciser monkey, 2017.

[31] A. Machiry, R. Tahiliani, M. Naik, Dynodroid: An input generation system for android apps, in: Proc. of the 9th Joint Meeting on Foundations of Software Engineering, ESEC/FSE, ACM, 2013, pp. 599-609.

[32] T. Su, G. Meng, Y. Chen, K. Wu, et al., Guided, stochastic model-based gui testing of android apps, in: Proc. 11th Joint Meeting on Foundations of Software Engineering, ESEC/FSE, ACM, 2017, pp. 245-256.

[33] H. B. Mann, D. R. Whitney, On a Test of Whether one of Two Random Variables is Stochastically Larger than the Other, The Annals of Mathematical Statistics 18 (1947) 50-60.

[34] A. Arcuri, L. Briand, A Hitchhiker's guide to statistical tests for assessing randomized algorithms in software engineering, Software Testing, Verification and Reliability 24 (2014) 219-250.

[35] F. G. de Oliveira Neto, R. Torkar, R. Feldt, L. Gren, C. A. Furia, Z. Huang, Evolution of statistical analysis in empirical software engineering research: Current state and steps forward, Journal of Systems and Software 156 (2019) 246-267. 
[36] A. Vargha, H. D. Delaney, A Critique and Improvement of the CL Common Language Effect Size Statistics of McGraw and Wong, Journal of Educational and Behavioral Statistics 25 (2000) 101-132.

[37] N. M. Albunian, Diversity in search-based unit test suite generation, in: Proc. of the 9th International Symposium on Search Based Software Engineering (SSBSE), Springer, 2017, pp. 183-189.

[38] G. Klees, A. Ruef, B. Cooper, S. Wei, M. Hicks, Evaluating fuzz testing, in: Proceedings of the 2018 ACM SIGSAC Conference on Computer and Communications Security, CCS '18, ACM, 2018, pp. 2123-2138.

[39] R. Jabbarvand, J. Lin, S. Malek, Search-based energy testing of android, in: Proceedings of the 41st International Conference on Software Engineering, ICSE, IEEE/ACM, 2019, pp. 1119-1130.

[40] W. Choi, G. C. Necula, K. Sen, Guided GUI testing of android apps with minimal restart and approximate learning, in: Proceedings of the International Conference on Object Oriented Programming Systems Languages \& Applications, OOPSLA, ACM, 2013, pp. 623-640.

[41] W. Song, X. Qian, J. Huang, Ehbdroid: beyond GUI testing for android applications, in: Proceedings of the 32nd International Conference on Automated Software Engineering, ASE, IEEE, 2017, pp. 27-37.

[42] S. Hao, B. Liu, S. Nath, W. G. Halfond, R. Govindan, Puma: Programmable ui-automation for large-scale dynamic analysis of mobile apps, in: Proc. of the 12th Int. Conference on Mobile Systems, Applications, and Services, MobiSys '14, ACM, 2014, pp. 204-217.

[43] R. Mahmood, N. Mirzaei, S. Malek, Evodroid: segmented evolutionary testing of android apps, in: Proc. of the 22nd International Symposium on Foundations of Software Engineering, FSE, ACM, 2014, pp. 599-609.

[44] Y. Li, Z. Yang, Y. Guo, X. Chen, Droidbot: a lightweight ui-guided test input generator for android, in: 39th International Conference on Software Engineering Companion (ICSE-C), IEEE, 2017, pp. 23-26.

[45] D. Amalfitano, A. R. Fasolino, P. Tramontana, B. D. Ta, A. Memon, Mobiguitar: Automated model-based testing of mobile apps, IEEE Softw. 32 (2015) 53-59.

[46] D. Amalfitano, V. Riccio, N. Amatucci, V. D. Simone, A. R. Fasolino, Combining automated GUI exploration of android apps with capture and replay through machine learning, Information \& Software Technology 105 (2019) 95-116. 
[47] T. Gu, C. Sun, X. Ma, C. Cao, C. Xu, Y. Yao, Q. Zhang, J. Lu, Z. Su, Practical GUI testing of android applications via model abstraction and refinement, in: Proceedings of the 41st International Conference on Software Engineering, ICSE, IEEE/ACM, 2019, pp. 269-280.

[48] Y. Li, Z. Yang, Y. Guo, X. Chen, Humanoid: A deep learning-based approach to automated black-box android app testing, in: Proc. of the 34th International Conference on Automated Software Engineering (ASE), IEEE, 2019, pp. 1070-1073.

[49] N. Mirzaei, S. Malek, C. S. Păsăreanu, N. Esfahani, R. Mahmood, Testing android apps through symbolic execution, Softw. Eng. Notes 37 (2012) 1-5.

[50] S. Yoo, M. Harman, Pareto efficient multi-objective test case selection, in: International Symposium on Software Testing and Analysis, ISSTA, ACM, 2007, pp. 140-150.

[51] M. Biagiola, A. Stocco, F. Ricca, P. Tonella, Diversity-based web test generation, in: Proceedings of the Joint Meeting on European Software Engineering Conference and Symposium on the Foundations of Software Engineering, ESEC/SIGSOFT FSE, ACM, 2019, pp. 142-153.

[52] R. Feldt, S. M. Poulding, Searching for test data with feature diversity, CoRR abs/1709.06017 (2017). arXiv: 1709.06017.

[53] R. Feldt, S. M. Poulding, Finding test data with specific properties via metaheuristic search, in: 24th International Symposium on Software Reliability Engineering, ISSRE, IEEE, 2013, pp. 350-359.

[54] Q. Shi, Z. Chen, C. Fang, Y. Feng, B. Xu, Measuring the diversity of a test set with distance entropy, IEEE Trans. Reliability 65 (2016) 19-27.

[55] F. G. de Oliveira Neto, R. Feldt, L. Erlenhov, J. B. de Souza Nunes, Visualizing test diversity to support test optimisation, in: 25th AsiaPacific Software Engineering Conference, IEEE, 2018, pp. 149-158.

[56] H. Waeselynck, P. Thévenod-Fosse, O. Abdellatif-Kaddour, Simulated annealing applied to test generation: Landscape characterization and stopping criteria, Empirical Softw. Engg. 12 (2007) 35-63.

[57] R. Lefticaru, F. Ipate, A comparative landscape analysis of fitness functions for search-based testing, in: 10th International Symposium on Symbolic and Numeric Algorithms for Scientific Computing, IEEE, 2008, pp. 201-208. 
[58] A. Aleti, I. Moser, L. Grunske, Analysing the fitness landscape of searchbased software testing problems, Automated Software Engineering 24 (2017) 603-621.

[59] N. Albunian, G. Fraser, D. Sudholt, Causes and effects of fitness landscapes in unit test generation, in: Proceedings of the Genetic and Evolutionary Computation Conference, GECCO '20, ACM, 2020, pp. 1204-1212.

[60] A. Aleti, I. Moser, Fitness landscape characterisation for constrained software architecture optimisation problems, in: 20th International Conference on Engineering of Complex Computer Systems (ICECCS), IEEE, 2015, pp. 11-20.

[61] G. Lu, R. Bahsoon, X. Yao, Applying elementary landscape analysis to search-based software engineering, in: 2nd International Symposium on Search Based Software Engineering, IEEE, 2010, pp. 3-8.

[62] F. Chicano, J. Ferrer, E. Alba, Elementary landscape decomposition of the test suite minimization problem, in: Search Based Software Engineering, Springer, 2011, pp. 48-63.

[63] C. Oliveira, A. Aleti, L. Grunske, K. Smith-Miles, Mapping the effectiveness of automated test suite generation techniques, IEEE Trans. Reliab. 67 (2018) 771-785. 